(C) 2000 Springer-Verlag New York Inc.

\title{
Approximating the Stance Map of a 2-DOF Monoped Runner
}

\author{
W. J. Schwind* and D. E. Koditschek ${ }^{\dagger}$ \\ Department of Electrical Engineering and Computer Science, The University of Michigan, \\ Ann Arbor, MI 48109-2110, USA \\ Received May 26, 1999; accepted January 6, 2000 \\ Online publication July 24, 2000 \\ Communicated by Stephen Wiggins
}

Summary. We report in this paper a relatively simple means of generating closed-form approximants to the return map associated with a family of nonintegrable Hamiltonian systems. These systems arise in consideration of legged locomotion by animals and robots. The approximations proceed through the iterated application of the mean value theorem for integral operators applied to a nonintegrable perturbation of the system of interest. Both the accuracy of these approximants and their algebraic intractability grow in a relatively controlled manner.

Key words. Running, central force, perturbation, approximation, Mean Value Theorem, Picard iterates, plant model, return map, Hamiltonian mechanics

MSC numbers. 37M99, 41A55, 68T40, 70E20, 70H07, 70K60

PAC numbers. 45.10.-b, 45.20.Jj, 45.40.Ln

\section{Introduction}

Natural locomotion systems rely on legs to gain tremendous advantage, relative to any present-day engineered vehicle, in traversing general terrain. The advantages of legs over wheels or treads are intuitively clear, but the field of robotics has been slow to develop synthetic systems that begin to approach the dexterity of even the humblest insect species. Legged robotic systems (excepting some very few pioneers to be dis-

\footnotetext{
* Supported in part by a National Science Foundation Graduate Research Fellowship and National Science Foundation Grant IRI-9612357.

$\dagger$ Supported in part by National Science Foundation Grant IRI-9510673.
} 


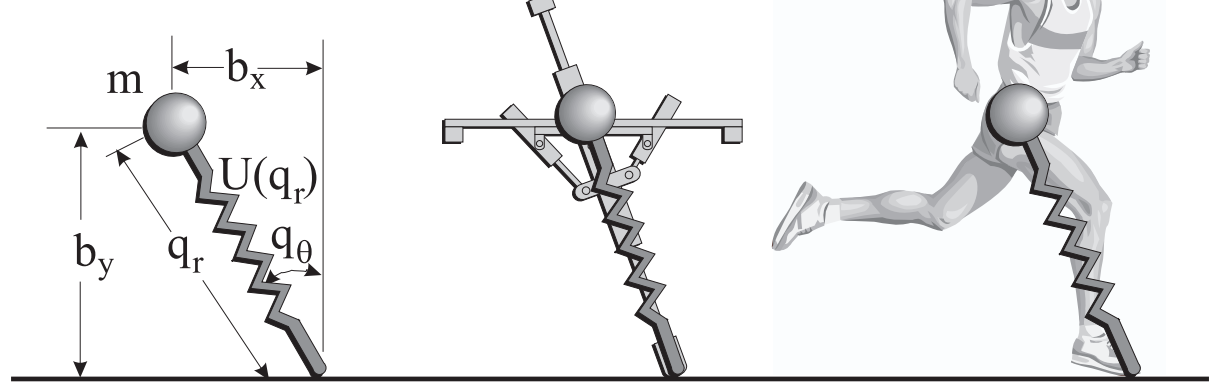

Fig. 1. (a) Left: The physical template: spring-loaded inverted pendulum (SLIP) monoped with point mass, $m$, at hip and massless leg consisting of a spring with potential $U\left(q_{r}\right)$. By convention, both $q_{\theta}$ and $b_{x}$ are defined to be negative to the left of vertical and positive to the right. (b) Middle: Illustration of the template's correspondence to Raibert's hopper. (c) Right: Illustration of the template's correspondence to a human runner.

cussed below [25], [32]) have been designed to operate in static [26] or quasi-static [30] stability. In contrast, animals seem to operate in the dynamical regime for all but the slowest of walks [10], [12]. In this paper, motivated by certain questions common to both robotics and biomechanics, we develop what may arguably be construed as the simplest possible "plant model"1 for general dynamical locomotion behavior in both robots and animals.

\subsection{Motivation: A Descriptively Complex, but Analytically Tractable Model}

That such a model would even exist may seem dubious. After all, legged animals present an incredible diversity of shape, size, and morphology. In spite of these obvious and important differences, biomechanists studying running ${ }^{2}$ [1], [4], [8], [10] have identified a striking underlying uniformity in the center of mass (COM) behavior of the majority of creatures studied. This seeming unity of strategy finds its archetype, or template, in the spring-loaded inverted pendulum (SLIP) depicted in Figure 1(a). The view of

\footnotetext{
${ }^{1}$ From the perspective of systems engineering, a particular physical phenomenon may be represented by either an input/output map or a full state internal model — the latter by linear or nonlinear dynamics; and these, in turn, may be cast in continuous or discrete time; and so on. In the end, the "correct" mathematical representation is dictated by the problem of concern and the available analysis and design tools. We will honor traditional usage by calling this representation the "plant model." The reader should beware that the term "plant" is accorded multiple meanings in the systems literature. It sometimes denotes the actual physical system, sometimes an idealized physical abstraction, and sometimes an appropriate mathematical representation. In this paper, we use the term "plant model" strictly in the latter sense.

${ }^{2}$ Running is not best defined by the presence or absence of a flight phase, as is commonly thought, but rather by the phase difference between a body's gravitational potential and forward kinetic energy during stance [2], [29]. For example, Full demonstrates that a cockroach, while never having a flight phase, almost always uses a running gait [11].
} 
animal dexterity as arising from the orbits of strategically tuned spring-loaded actuators dates back to Bernstein [3], and contemporary biomechanists have posited the SLIP as a specific template for fast legged locomotion behavior [4], [5], [10], [28]. Specifically, Full has presented strong evidence suggesting that animals, whether using two, four, six, eight or more legs, arrange their nerves and muscles in a manner that persuades their COM it is riding on such a pogo stick [10]. This notion of a "virtual SLIP" is suggested in Figure 1(c).

The utility of dynamical legged locomotion was introduced to robotics by Raibert [32]. His machines were designed to be explicitly SLIP-like, incorporating a physically identifiable spring connecting the toe to the body. The SLIP mechanics afforded simple yet ground-breaking strategies that relied on the shaping of total energy and reverse time symmetries for control. These strategies yielded stunning results for the planar monoped depicted in Figure 1(b), and were extended with equal success to an entire family of one-, two-, and four-legged machines running in three-dimensional space, again using intuitive notions of a "virtual SLIP" as suggested in Figure 1(c).

\subsection{Application: Control and Identification Schemes for Legged Locomotion}

Even subsequent to Raibert's pioneering designs, standard approaches to the control of legged locomotion have continued to employ feedback controllers to track joint space reference trajectories [23], [42], [45], [46]. A fundamental question arises regarding the origin of these reference signals. Since translation of desired body motions into joint space trajectories that achieve them represents a central unknown, a variety of different approaches have been proposed. In character animation, for example, these signals are often generated via playback of motion capture recordings from animals [13] or from interpolation of tediously assigned key frames [9]. In robotics, reference trajectories are often derived by minimization of an energy or torque cost function [6], [34]. Each of these previous techniques requires storage of a library of reference motions which must be derived off-line. Obviously such libraries will not be complete, and motions not represented must be interpolated from the others. Other approaches avoid this problem by implicitly encoding the desired motion either in terms of constraint equations [20], [21], [17], [16] or in terms of simpler dynamical systems [7], [30], [32], [36]. In any case, from our perspective, it seems that for control of the body using the legs, one should seek analytically tractable principles that can be asserted in terms of high-level control commands (or gait parameters) such as "run this fast" or "jump this high" and which can be easily extended to runners with varying morphology [36].

In the case of animals, one wishes to test from motion capture data how closely the COM trajectory matches SLIP orbits [37], characterize the potential law of the effective virtual spring [37], trace back the contribution of the physical joint torques toward this overall target, and begin to hypothesize about the muscle recruitment strategies that might achieve it. This is a problem (at least in the beginning stages) of statistical parameter estimation. Identification, however, demands not merely a conveniently parametrized family of appropriate potential functions but a view of how any specific instance manifests itself in the resulting orbits. 

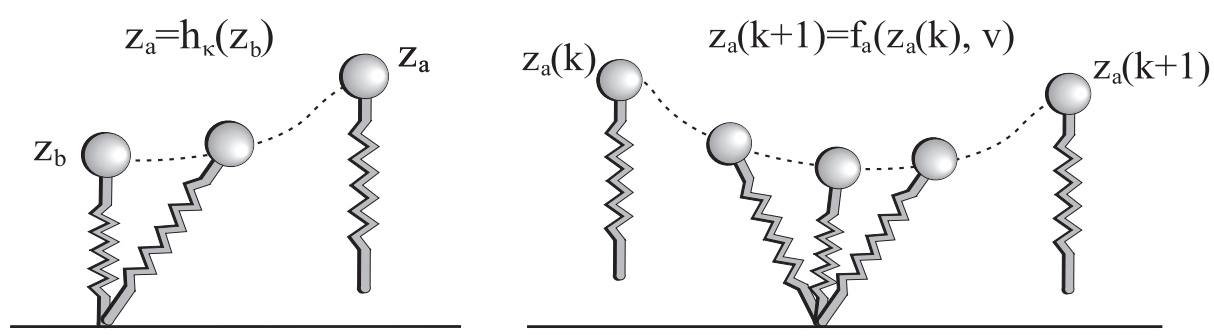

Fig. 2. Bottom to apex map (left) and apex return map (right).

For both control and estimation, the established techniques of systems engineering presume a parsimoniously parametrized plant model. This forces the sharply revised question of whether such a complex physical model as the SLIP can be used to generate a plant model suitable for legged locomotion? Addressing that question constitutes the central burden of our paper.

\subsection{Scope: The Parametrized Return Map as Plant Model}

For the stated purposes of control and estimation, the continuous time COM trajectories of the SLIP leg do not represent an effective plant model. To begin with, this is a hybrid dynamical problem. There are really two vector fields at play, since many runners alternate between stance and flight modes. Moreover, as we have argued above, the task of locomotion, while built on the orbits of the underlying Hamiltonian vector field, is more naturally encoded in the abstract terms of heights and speeds and duty factors. Finally, from the perspective of control authority, synthetic actuators do not possess sufficient power density and natural proprioception likely does not provide sufficient bandwidth to afford the traditional control theorist's reliance on feedforward/feedback techniques of trajectory tracking in the physical application settings of interest. It makes much more sense to think in terms of the discrete time maps depicted in Figure 2 that relate the runner's states at bottom and apex of a trajectory. In this formulation, the plant model arises as a "controlled return map" whose states represent the elements of a Poincaré section transverse to the periodic orbits of running and whose inputs represent the integrated effect of certain parameter settings - the ones representing all actuator activity that transpires over a given cycle.

This approach introduces an obvious new difficulty. The Hamiltonian vector field that generates the SLIP COM trajectories takes the general form of the restricted threebody problem whose nonintegrability was established a century ago by Poincaré [19]. In other words, our presumed plant model cannot even be written down. However, in our experience, for purposes of systems engineering (i.e., both from the controls and the identification points of view), plant models need to be written down in some conveniently parametrized closed form. Numerical simulation can be used to gain human insight into the salient features of the physical setting. But designing realtime feedback control laws - at least any sort whose effects are to be predictably reliableinvolves devising policies that are functionally related to the plant model. Further- 
more, implementing such policies involves the use of computational data structures whose entries are functionally related to calibrated instances of the plant model parameters. Similarly, parameter identification - at least any sort that can entail empirically refutable hypotheses - involves fitting parsimonious functional representations to limited observed behavior with the object of making automated predictions about the future behavior.

In short, some representation of the plant must be available in closed form. But for the phenomena of interest, the plant model amounts to the return map of a nonintegrable dynamical system and, hence, seems to defy the possibility of representation.

\subsection{Contribution: Approximations through Iterated Application of the Mean Value Theorem for Integrals}

This impasse motivates the central problem addressed in this paper. We seek a close approximation to the stance map of the SLIP leg that can be written as a closed-form function of its Hamiltonian vector field. How close is close? Our somewhat pragmatic attitude is that this depends upon the application. For purposes of control, particularly when relatively high authority feedback is possible, plant models that capture more than ninety percent of the systematic variation in a physical process may suffice. In contrast, for purposes of estimation, when each step - the sensor noise floor, uncontrolled sources of process variation, and so on-contributes to the residual error, one seeks absolutely the most accurate possible useful representation of the systematic variations that preserves the key physical properties. Finally, our interest in pursuing the SLIP as a template for higher degree of freedom systems [36] argues for as great an accuracy as possible. We report in this paper a relatively simple means of generating closedform approximants whose accuracy and algebraic intractability both grow in a relatively controlled manner through the iterated application of the mean value theorem for integral operators.

It is natural to inquire how well any method of approximation handles qualitative changes in the object being approximated. Unfortunately, the formal view of "qualitative difference" in the setting of dynamical systems has an asymptotic character (e.g., has the topology of some limit set changed?) while we are only concerned with the short-term behavior of the underlying continuous time dynamics. For example, because our approximants assume a Hamiltonian system and because no Hamiltonian system is structurally stable, there is no reason to expect that our approach will yield useful asymptotic results when friction is present. To the contrary, however, in one degree of freedom settings (where everything is integrable, so approximation is not at issue), apex return maps of the sort depicted in Figure 2 derived from Hamiltonian models have been used to stand in for the behavior of lossy hopping [22] and juggling [33] with very favorable physical outcomes - arguably because the short-term behaviors of the "qualitatively different" systems are quite similar. Abandoning formal notions of "qualitative difference," we take the more utilitarian approach of presenting statistical summaries of numerical comparisons between simulated orbits (the presumed "truth") and various parametrically different models. 
A feeling for the nature of these results is provided by a glance at the function

$$
h_{U}^{1}\left(q_{r}\right)=\left[\begin{array}{c}
\hat{t}_{s 1}\left(q_{r}\right) \\
\hat{q}_{\theta 1}\left(q_{r}\right) \\
\hat{p}_{\theta 1}\left(q_{r}\right) \\
\hat{p}_{r_{1}}\left(q_{r}\right)
\end{array}\right]=\left[\begin{array}{c}
t_{s b}+\frac{m}{H_{p e r t}^{-1}\left(\hat{\xi}_{r}, \hat{q}_{\theta 0}\left(\hat{\xi}_{r}\right), \hat{p}_{\theta 0}\left(\hat{\xi}_{r}\right)\right)}\left(q_{r}-r_{b}\right) \\
\theta_{b}+\frac{\hat{p}_{\theta 0}\left(\hat{\xi}_{r}\right)}{\hat{\xi}_{r}^{2} H_{p e r t}^{-1}\left(\hat{\xi}_{r}, \hat{q}_{\theta 0}\left(\hat{\xi}_{r}\right), \hat{p}_{\theta 0}\left(\hat{\xi}_{r}\right)\right)}\left(q_{r}-r_{b}\right) \\
p_{\theta b}+\frac{m^{2} g \hat{\xi}_{r} \sin \left(\hat{q}_{\theta 0}\left(\hat{\xi}_{r}\right)\right)}{H_{\text {pert }}^{-1}\left(\hat{\xi}_{r}, \hat{q}_{\theta 0}\left(\hat{\xi}_{r}\right) \hat{p}_{\theta 0}\left(\hat{\xi}_{r}\right)\right)}\left(q_{r}-r_{b}\right) \\
H_{p e r t}^{-1}\left(q_{r}, \hat{q}_{\theta 1}\left(q_{r}\right), \hat{p}_{\theta 1}\left(q_{r}\right)\right)
\end{array}\right],
$$

which we obtain by applying our approximation technique to the Hamiltonian vector field,

$$
X_{H_{p e r t}}(\boldsymbol{q}, \boldsymbol{p})=\left[\begin{array}{c}
\frac{p_{r}}{m} \\
\frac{p_{\theta}}{m q_{r}^{2}} \\
\frac{p_{\theta}^{2}}{m q_{r}^{3}}-D U\left(q_{r}\right) \\
0
\end{array}\right]+\left[\begin{array}{c}
0 \\
0 \\
-m g \cos \left(q_{\theta}\right) \\
m g q_{r} \sin \left(q_{\theta}\right)
\end{array}\right],
$$

arising from the SLIP of Figure 1(a) forced by a spring with the potential energy function $U\left(q_{r}\right)$. Here

$$
\begin{aligned}
& H_{\text {pert }}^{-1}\left(q_{r}, q_{\theta}, p_{\theta}\right)=[ 2 m\left[\left(U\left(r_{b}\right)-U\left(q_{r}\right)\right)+m g\left(r_{b} \cos \left(\theta_{b}\right)-q_{r} \cos \left(q_{\theta}\right)\right)\right] \\
&\left.+\left(\frac{p_{\theta b}^{2}}{r_{b}^{2}}-\frac{p_{\theta}^{2}}{q_{r}^{2}}\right)\right]^{\frac{1}{2}},
\end{aligned}
$$

and $\hat{\xi}_{r}=(1 / 4) q_{r}+(3 / 4) r_{b}$. Additionally, the numerical subscript identifies the particular iterate - both $\hat{q}_{\theta 0}$ and $\hat{p}_{\theta_{0}}$ are defined in (2). Other notations are defined in Table 1.

Although $X_{H_{p e r t}}$ is nonintegrable and the spring potential, $U$, is not prescribed at all, $h_{U}^{1}$ closely approximates the results of numerically integrating trajectories of $X_{H_{p e r t}}$ from stance bottom states (where the leg spring is maximally compressed) to flight apex states (where the body's vertical height is greatest) for all instances we have examined when $U$ is a convex function (i.e., the spring resists compression with a nonnegative stiffness). For example, when we take the potential function of (47),

$$
U_{T H K}\left(q_{r}\right)=\frac{k}{2}\left(\arccos \left[\frac{q_{r}^{2}-l_{1}^{2}-l_{2}^{2}}{2 l_{1} l_{2}}\right]-\arccos \left[\frac{q_{r l}^{2}-l_{1}^{2}-l_{2}^{2}}{2 l_{1} l_{2}}\right]\right)^{2},
$$

resulting from a torsional Hooke's law spring at the "knee" of the slightly more zoomorphic leg (see Appendix B) of Figure 8, we produce with (1) and (2) a map that takes the cubic cell of bottom states shown in Figure 7(a) into a twisted volume of apex states similar to that of Figure 7(b). The bottom state cube of Figure 7(a) contains leg lengths in the range $r_{b} \in[0.75,0.975] \mathrm{m}$, angular momentum in the range $p_{\theta b} \in[1.5,6.5] \mathrm{kg} \mathrm{m} \mathrm{m}^{2} \mathrm{rad} / \mathrm{s}$, and spring potential energy in the range $U\left(r_{b}\right) \in[2.5,7.5] \mathrm{kg} \mathrm{m} / \mathrm{s}^{2}$, and we shall use it 
throughout the paper as a means of comparing approximants, since it results in a typical range of human gaits: hopping heights in the range $y_{a} \in[0.77,1.74] \mathrm{m}$, forward velocities in the range $\dot{x}_{a} \in[1.56,9.26] \mathrm{m} / \mathrm{s}$, and duty factors in the range $\beta \in[0.01,0.41]$, where we assume a leg length of $1 \mathrm{~m}$ at liftoff and a mass of $1 \mathrm{~kg}$.

To characterize the quality of an approximant, such as $h_{U}^{1}$, we compare its image to the result of numerically integrating $X_{H_{p e r t}}$ over this entire cube. We report the average and maximum percent errors and the standard deviations for a number of relevant state variables, as shown in Figure 6. We will find it convenient to report all such numerical comparisons in a similar format throughout the paper. For instance, computing the leg angle at liftoff, $q_{\theta l}$, using $h_{U}^{1}$ incurs a mean error of $3.25 \%$, with standard deviation of $1.46 \%$ and maximum error of $14.6 \%$.

According to the reasoning above, we may, depending upon the application, prefer instead of $h_{U}^{1}$ a less accurate approximant with a simpler closed-form representation or a more accurate approximant involving correspondingly more complicated terms. Trading off accuracy for functional simplicity is achieved in our approximation technique by the iteration number of the functional composition procedure detailed in Section 4. For example, the zeroth iterate of this procedure is given by

$$
h_{U}^{0}\left(q_{r}\right)=\left[\begin{array}{c}
\hat{t}_{s_{0}}\left(q_{r}\right) \\
\hat{q}_{\theta_{0}}\left(q_{r}\right) \\
\hat{p}_{\theta_{0}}\left(q_{r}\right) \\
\hat{p}_{r_{0}}\left(q_{r}\right)
\end{array}\right]=\left[\begin{array}{c}
\frac{m\left(q_{r}-r_{b}\right)}{\left[p_{\theta b}^{2}\left(\frac{1}{r_{b}^{2}}-\frac{1}{\hat{\xi}_{r}^{2}}\right)+2 m\left(U\left(r_{b}\right)-U\left(\hat{\xi}_{r}\right)\right)\right]^{\frac{1}{2}}} \\
\frac{p_{\theta b}\left(q_{r}-r_{b}\right)}{\hat{\xi}_{r}^{2}\left[p_{\theta b}^{2}\left(\frac{1}{r_{b}^{2}}-\frac{1}{\xi_{r}^{2}}\right)+2 m\left(U\left(r_{b}\right)-U\left(\hat{\xi}_{r}\right)\right)\right]^{\frac{1}{2}}} \\
p_{\theta b} \\
{\left[p_{\theta b}^{2}\left(\frac{1}{r_{b}^{2}}-\frac{1}{q_{r}^{2}}\right)+2 m\left(U\left(r_{b}\right)-U\left(q_{r}\right)\right)\right]^{\frac{1}{2}}}
\end{array}\right]
$$

and results in an approximation accuracy yielding mean error of $7.11 \%$ with standard deviation of $3.74 \%$ and maximum error of $30.9 \%$ for $q_{\theta l}$. The approximant previously examined, $h_{U}^{1}$, results from one application of the iterative procedure. In contrast, the second iterate, which takes the same form as (1) except with the subscript 1 replaced with the subscript 2 and the subscript 0 replaced with the subscript 1 , results in an approximation accuracy yielding mean error of $1.51 \%$ with standard deviation of $1.04 \%$ and maximum error of $7.01 \%$.

\subsection{Organization of the Paper}

The SLIP monoped operates in two distinct dynamical phases, depending on whether it is on the ground or in the air. Section 2 introduces the Hamiltonian dynamics of both phases.

The first question addressed is the importance of the spring law. While virtually all successful running robots to date have adopted the revolute-prismatic kinematics of the SLIP monoped, biomechanists have heretofore adopted this model [5] only in analogy to the more biologically valid revolute-revolute kinematics, the toe and knee depicted in Figure 8. Thus, while it is straightforward to express a given spring law in one or another set of coordinates, it is equally clear that simple expressions in one set will yield 
very complex expressions in the other, and vice versa. More fundamentally, actuation technology in robotics is incredibly diverse, and the form and function of animal muscles is similarly varied. We seek a mode of analysis that does not commit to any specific spring form. Any physically interesting SLIP spring potential law must be repulsive in nature. That is, its force must increase with increased leg compression. While a multitude of such springs could be proposed, certain special cases yield solvable elliptic integrals in the completely integrable setting when there is no gravity during stance (a case we refer to as the unperturbed SLIP). Might such an exemplar do a "good enough" job of capturing the behavior of other springs? Simulation evidence presented in Section 3 demonstrates that the particular functional form of the spring law is important, especially for predicting quantities such as stance time and duty factor.

Motivated by this observation, we introduce a means of generating closed-form approximants for the unperturbed stance dynamics for arbitrary spring laws. This result relies on a novel application of the Mean Value Theorem for Integrals. Simulation data is presented for the adequacy of this approach in the unperturbed setting.

After removing the restriction on the functional form of the spring, it remains to address the absence of gravity during stance. In most previous robotics research, gravity has been ignored during stance, with the presumption that the spring contributes the dominant radial force and that the angular momentum about the toe is almost constant [22], [27], [39], [43]. Simulation results presented in Section 4 demonstrate that gravity cannot be ignored in stance if a wide range of orbits is desired. We thus introduce an iteration procedure, combining Picard style iterates with the Mean Value Theorem for Integrals result of Section 3, to construct approximate solutions to the perturbed dynamics whose accuracy and complexity grow in a controlled manner as exemplified in equations (1) and (2). Again, simulation data is compared to establish the efficacy of this approach.

\section{The SLIP Template}

\subsection{Notation and Terminology}

The SLIP template, depicted in Figure 1(a), is defined according to the following assumptions. The leg is assumed to be a massless spring, with potential law $U\left(q_{r}\right)$, that connects the toe to a point mass, $m$, at the hip. It is assumed that there are no losses during stance or flight and that the only force acting during flight is gravity. Furthermore, it is assumed that once on the ground the toe does not slip, effectively acting as a hinge about which the leg is free to rotate in the sagittal plane.

The hopping cycle consists of two primary phases: the stance phase, when the foot is on the ground, and the flight phase, when the leg is airborne. The stance phase can be further decomposed into two subphases: compression and decompression. ${ }^{3}$ Four important events must occur during one hopping cycle: Touchdown, the moment the foot makes contact with the ground; Bottom, the moment during stance when the robot reaches

\footnotetext{
${ }^{3}$ A third subphase is often introduced - the thrust phase [22], [32], [43]. For purposes of this paper, it suffices to assume that any thrust occurs instantaneously.
} 
Table 1. Notation used throughout the paper.

\begin{tabular}{|c|c|}
\hline \multicolumn{2}{|r|}{ Leg Coordinates } \\
\hline$q_{r}$ & Leg Length \\
\hline$q_{\theta}$ & Leg Angle from Vertical \\
\hline$p_{r}$ & Radial Momentum \\
\hline$p_{\theta}$ & Angular Momentum \\
\hline$b_{x}$ & Horizontal Position of Body \\
\hline$b_{y}$ & Vertical Position of Body \\
\hline$p_{x}$ & Horizontal Momentum \\
\hline$p_{y}$ & Vertical Momentum \\
\hline $\boldsymbol{b}$ & Cartesian Position Coordinates, $\left[b_{x}, b_{y}\right]^{T}$ \\
\hline$b$ & Cartesian Velocity Coordinates, $\left[\dot{b}_{x}, \dot{b}_{y}\right]^{T}$ \\
\hline$p_{b}$ & Cartesian Momentum Coordinates, $\left[p_{x}, p_{y}\right]^{T}$ \\
\hline$T \boldsymbol{b}$ & {$[\boldsymbol{b}, \dot{\boldsymbol{b}}]^{T}$} \\
\hline$T^{*} \boldsymbol{b}$ & {$\left[\boldsymbol{b}, \boldsymbol{p}_{\boldsymbol{b}}\right]^{T}$} \\
\hline$q$ & Polar Position Coordinates, $\left[q_{r}, q_{\theta}\right]^{T}$ \\
\hline$\dot{\boldsymbol{q}}$ & Polar Velocity Coordinates $\left[\dot{q}_{r}, \dot{q}_{\theta}\right]^{T}$ \\
\hline$p_{q}$ & Polar Momentum Coordinates, $\left[p_{r}, p_{\theta}\right]^{T}$ \\
\hline$T q$ & {$[\boldsymbol{q}, \dot{\boldsymbol{q}}]^{T}$} \\
\hline$T^{*} \boldsymbol{q}$ & {$\left[\boldsymbol{q}, \boldsymbol{p}_{\boldsymbol{q}}\right]^{T}$} \\
\hline \multicolumn{2}{|r|}{ Bottom Parameters } \\
\hline$r_{b}$ & Leg Length at Bottom \\
\hline$\theta_{b}$ & Leg Angle at Bottom \\
\hline$p_{\theta b}$ & Angular Momentum at Bottom \\
\hline$k$ & Spring Constant \\
\hline \multicolumn{2}{|r|}{ Liftoff Parameters } \\
\hline$q_{r l}$ & Leg Length at Liftoff \\
\hline$q_{\theta l}$ & Leg Angle at Liftoff \\
\hline$p_{r l}$ & Radial Momentum at Liftoff \\
\hline$p_{\theta l}$ & Angular Momentum at Liftoff \\
\hline$\dot{b}_{x l}$ & Forward Velocity at Liftoff \\
\hline$\dot{b}_{y l}$ & Vertical Velocity at Liftoff \\
\hline$t_{s}$ & Stance Time \\
\hline \multicolumn{2}{|r|}{ Apex Parameters } \\
\hline$y_{a}$ & Apex Hopping Height \\
\hline$\dot{x}_{a}$ & Apex Forward Velocity \\
\hline$t_{f}$ & Flight Time \\
\hline$\beta$ & Duty Factor $=\frac{t_{s}}{2\left(t_{s}+t_{f}\right)}$ \\
\hline \multicolumn{2}{|r|}{ Dynamical Parameters } \\
\hline$m$ & Body Mass \\
\hline$g$ & Acceleration due to Gravity \\
\hline
\end{tabular}

maximal compression and the radial momentum changes from inward to outward; Liftoff, the moment the foot loses contact with the ground; and Apex, the moment in the flight phase where the robot has maximum amplitude and vertical motion changes from upward to downward.

Some further notational conventions are presented in Table 1. 


\subsection{The SLIP Dynamics}

The Hamiltonian vector fields for both stance and flight phases take the following form.

Stance Dynamics. Using the notation of Table 1, we can write the Hamiltonian (total energy) in the following convenient form,

$$
H_{\text {pert }}=H_{\text {unpert }}+H_{\text {grav }}
$$

where

$$
H_{\text {unpert }}=\frac{1}{2 m}\left(p_{r}^{2}+\frac{p_{\theta}^{2}}{q_{r}^{2}}\right)+U\left(q_{r}\right) \quad \text { and } \quad H_{\text {grav }}=m g q_{r} \cos q_{\theta} .
$$

Note that $H_{\text {unpert }}$ is the sum of the kinetic and spring potential energies, while $H_{\text {grav }}$ is simply the gravitational potential energy.

The vector field, $X_{H_{\text {unpert }}}$ arising from the Hamiltonian, $H_{\text {unpert }}$ is given by

$$
\left[\begin{array}{c}
\dot{q}_{r} \\
\dot{q}_{\theta} \\
\dot{p}_{r} \\
\dot{p}_{\theta}
\end{array}\right]=X_{H_{\text {unpert }}}(\boldsymbol{q}, \boldsymbol{p})=\left[\begin{array}{c}
\frac{p_{r}}{m} \\
\frac{p_{\theta}}{m q_{r}^{2}} \\
\frac{p_{\theta}^{2}}{m q_{r}^{3}}-D U\left(q_{r}\right) \\
0
\end{array}\right]
$$

Notice that these are the dynamics of a classic central force problem. There are two integral invariants, and the system is completely integrable [14], [39], [44].

The vector field arising from $H_{\text {grav }}$ is given by

$$
\left[\begin{array}{c}
\dot{q}_{r} \\
\dot{q}_{\theta} \\
\dot{p}_{r} \\
\dot{p}_{\theta}
\end{array}\right]=X_{H_{g r a v}}(\boldsymbol{q}, \boldsymbol{p})\left[\begin{array}{c}
0 \\
0 \\
-m g \cos \left(q_{\theta}\right) \\
m g q_{r} \sin \left(q_{\theta}\right)
\end{array}\right]
$$

and when added to that of (4) destroys the $q_{\theta}$ symmetry, resulting in a nonintegrable problem.

Although (4) will be referred to as the unperturbed dynamics and (4) with the addition of (5) as the perturbed dynamics, in contrast to traditional perturbation methods, we do not impose the restriction that the perturbation is "sufficiently" small.

Flight Dynamics. As the leg decompresses during the stance phase, it reaches a point where the rising hip pulls the leg off the ground and the flight phase begins. At this point it is convenient to change from polar (stance, $\boldsymbol{q}=\left[q_{r}, q_{\theta}\right]^{T}, \boldsymbol{p}_{\boldsymbol{q}}=\left[p_{r}, p_{\theta}\right]^{T}$ ) to cartesian 
(flight, $\boldsymbol{b}=\left[b_{x}, b_{y}\right]^{T}, \boldsymbol{p}_{\boldsymbol{b}}=\left[p_{x}, p_{y}\right]^{T}$ ) coordinates using the following relationships: ${ }^{4}$

$$
\boldsymbol{b}=g(\boldsymbol{q})=\left[\begin{array}{l}
q_{r} \sin q_{\theta} \\
q_{r} \cos q_{\theta}
\end{array}\right]
$$

and $^{5}$

where

$$
\boldsymbol{p}_{\boldsymbol{b}}=M_{B} D g M_{Q}^{-1} \boldsymbol{p}_{\boldsymbol{q}}
$$

$$
M_{B}=\left[\begin{array}{cc}
m & 0 \\
0 & m
\end{array}\right], \quad M_{Q}=\left[\begin{array}{cc}
m & 0 \\
0 & m q_{r}^{2}
\end{array}\right], \quad \text { and } \quad D g=\left[\begin{array}{cc}
\sin q_{\theta} & q_{r} \cos q_{\theta} \\
\cos q_{\theta} & -q_{r} \sin q_{\theta}
\end{array}\right] .
$$

The monoped flies through the air as a two degree-of-freedom point mass subject to gravity, until the leg touches down again. We assume that the leg angle at touchdown can be freely selected in flight. For such a model, the Hamiltonian can be written as

$$
H_{F}=\frac{1}{2 m}\left(p_{x}^{2}+p_{y}^{2}\right)+m g b_{y} .
$$

Then the vector field, $X_{H_{F}}$, arising from the Hamiltonian, $H_{F}$, is given by

$$
\left[\begin{array}{c}
\dot{b}_{x} \\
\dot{b}_{y} \\
\dot{p}_{x} \\
\dot{p}_{y}
\end{array}\right]=X_{H_{F}}(\boldsymbol{b}, \boldsymbol{p})=\left[\begin{array}{c}
\frac{p_{x}}{m_{y}} \\
\frac{p_{y}}{m} \\
0 \\
-m g
\end{array}\right] .
$$

\section{Integrating the Unperturbed Stance Dynamics}

\subsection{Integration by Quadratures}

Newtonian free flight dynamics (10) are readily integrable, so the only point of inquiry concerns the stance dynamics. As mentioned above, the vector field $X_{H_{\text {unpert }}}$ (4) corresponding to the unperturbed system is completely integrable-having two constants of motion: the total energy, $E_{0}=H_{\text {unpert }}$, and the angular momentum, $L_{0}$. Integrating by quadratures, ${ }^{6}$ we use constant $L_{0}$ to give $p_{\theta}$ and this, in addition to constant $E_{0}$, to give $p_{r}$,

$$
\begin{aligned}
& p_{\theta}\left(q_{r}\right)=L_{0} \\
& p_{r}\left(q_{r}\right)=\left[2 m\left(E_{0}-U\left(q_{r}\right)\right)-\frac{L_{0}^{2}}{q_{r}^{2}}\right]^{\frac{1}{2}} .
\end{aligned}
$$

\footnotetext{
${ }^{4}$ Here, both $q_{\theta}$ and $b_{x}$ are defined to be negative to the left of vertical and positive to the right, as defined in Figure 1(a).

${ }^{5}$ We denote the jacobian of $g$ and all other functions by the symbol $D g$.

${ }^{6}$ Choosing $q_{r}$ as the dependent variable conforms to tradition [14, §3-5] [24, §1.3c] [44, §48], suits well the need to leave unspecified the spring law $U\left(q_{r}\right)$, and easily allows the convention of characterizing the transition from stance to flight by the achievement of a particular leg length, $q_{r}=q_{r l}$ [22], [27], [32], [39], [43].
} 
With no loss of generality, we focus attention on the decompression phase $\left(p_{r}>0\right)$ in this work and, therefore, select the positive square root solution for $p_{r}$. With the results of (11), the remaining (elliptic) integrals are given by

$$
\begin{aligned}
& q_{\theta}\left(q_{r}\right)=\theta_{b}+\int_{r_{b}}^{q_{r}} \frac{p_{\theta}}{\rho^{2} p_{r}(\rho)} d \rho=\theta_{b}+\int_{r_{b}}^{q_{r}} \frac{L_{0}}{\rho^{2}\left[2 m\left(E_{0}-U(\rho)\right)-\frac{L_{0}^{2}}{\rho^{2}}\right]^{\frac{1}{2}}} d \rho, \\
& t_{s}\left(q_{r}\right)=t_{s b}+\int_{r_{b}}^{q_{r}} \frac{m}{p_{r}(\rho)} d \rho=t_{s b}+\int_{r_{b}}^{q_{r}} \frac{m}{\left[2 m\left(E_{0}-U(\rho)\right)-\frac{L_{0}^{2}}{\rho^{2}}\right]^{\frac{1}{2}}} d \rho,
\end{aligned}
$$

where the initial condition (the bottom state, since we are focusing on decompression) is $\left[r_{b}, \theta_{b}, 0, p_{\theta b}\right]^{T}$. Substituting the angular momentum and the energy at bottom, $L_{0}=p_{\theta b}$ and $E_{0}=\frac{1}{2 m} \frac{p_{\theta b}^{2}}{r_{b}^{2}}+U\left(r_{b}\right)$, we find

$$
\begin{aligned}
& p_{\theta}\left(q_{r}\right)=p_{\theta b}, \\
& p_{r}\left(q_{r}\right)=\left[p_{\theta b}^{2}\left(\frac{1}{r_{b}^{2}}-\frac{1}{q_{r}^{2}}\right)+2 m\left(U\left(r_{b}\right)-U\left(q_{r}\right)\right)\right]^{\frac{1}{2}}, \\
& q_{\theta}\left(q_{r}\right)=\theta_{b}+\int_{r_{b}}^{q_{r}} \frac{p_{\theta b}}{\rho^{2}\left[p_{\theta b}^{2}\left(\frac{1}{r_{b}^{2}}-\frac{1}{\rho^{2}}\right)+2 m\left(U\left(r_{b}\right)-U(\rho)\right)\right]^{\frac{1}{2}}} d \rho, \\
& t_{s}\left(q_{r}\right)=t_{s b}+\int_{r_{b}}^{q_{r}} \frac{m}{\left[p_{\theta b}^{2}\left(\frac{1}{r_{b}^{2}}-\frac{1}{\rho^{2}}\right)+2 m\left(U\left(r_{b}\right)-U(\rho)\right)\right]^{\frac{1}{2}}} d \rho .
\end{aligned}
$$

\subsection{Exact Solution for an Air Spring}

While the equations of motion for the unperturbed system are integrable in the formal mathematical sense (13), the resulting elliptic integrals are almost as opaque to the kind of parametric insight desired in our applications as Runge-Kutta simulations would be. Indeed, for a general spring potential, the integrals given in (13) cannot be written in terms of elementary functions.

Whittaker [44, \$48] exhibits a small class of spring laws for which the central force problem can be integrated in terms of elementary functions. One in particular,

$$
U_{A}\left(q_{r}\right)=\frac{k}{2}\left(\frac{1}{q_{r}^{2}}-\frac{1}{q_{r l}^{2}}\right) \quad \text { and } \quad D U_{A}\left(q_{r}\right)=-\frac{k}{q_{r}^{3}},
$$

offers the virtue of a "simple" closed-form solution while also providing a simplistic, but not unreasonable, model of the compressed air spring that Raibert used in many of his robots [22], [32], [39]. For this reason, throughout the remainder of the paper we will refer to this spring as the Air Spring.

For this choice, the integrals of (13) can be written in terms of elementary functions as

$$
\begin{aligned}
& p_{\theta}\left(q_{r}\right)=p_{\theta b}, \\
& p_{r}\left(q_{r}\right)=\left[\frac{\left(p_{\theta b}^{2}+m k\right)\left(q_{r}^{2}-r_{b}^{2}\right)}{q_{r}^{2} r_{b}^{2}}\right]^{\frac{1}{2}},
\end{aligned}
$$




$$
\begin{aligned}
& q_{\theta}\left(q_{r}\right)=\theta_{b}+\frac{p_{\theta b}}{\sqrt{p_{\theta b}^{2}+m k}} \operatorname{arccot}\left[\sqrt{\frac{r_{b}^{2}}{q_{r}^{2}-r_{b}^{2}}}\right], \\
& t_{s}\left(q_{r}\right)=t_{s b}+m r_{b} \sqrt{\frac{q_{r}^{2} r_{b}^{2}}{p_{\theta b}^{2}+m k}} .
\end{aligned}
$$

In the introduction we briefly discussed the importance of the spring law. It is natural to ask at this point whether such concern is justified. In other words, could we use the closed-form Air Spring solution given in (15) as an approximant to the solution of the integrated stance dynamics (13) for other spring laws?

This question is more than academic, since other spring laws appear prominently in the running literature. For example, the familiar Hooke's law spring is used extensively by the biomechanists in their running studies [4], [5], [8], and it also accurately models the springs used in Buehler's running machines [15], [31].

In an attempt to address this question, we introduce two different spring models, a SLIP Hooke's Law Spring,

$$
U_{H}\left(q_{r}\right)=\frac{k}{2}\left(r_{l}-q_{r}\right)^{2} \quad \text { and } \quad D U_{H}\left(q_{r}\right)=-k\left(r_{l}-q_{r}\right),
$$

and a "Torsional Hooke's Law" pulled back from the "knee" of the revolute-revolute leg shown in Figure 8. The potential and force law for this spring, which are derived more thoroughly in Appendix B, are given by

$$
\begin{aligned}
U_{T H K}\left(q_{r}\right) & =\frac{k}{2}\left(\arccos \left[\frac{q_{r}^{2}-l_{1}^{2}-l_{2}^{2}}{2 l_{1} l_{2}}\right]-\arccos \left[\frac{q_{r l}^{2}-l_{1}^{2}-l_{2}^{2}}{2 l_{1} l_{2}}\right]\right)^{2} \quad \text { and } \\
D U_{T H K}\left(q_{r}\right) & =-2 q_{r} k \frac{\left(\arccos \left[\frac{q_{r}^{2}-l_{1}^{2}-l_{2}^{2}}{2 l_{1} l_{2}}\right]-\arccos \left[\frac{q_{r l}^{2}-l_{1}^{2}-l_{2}^{2}}{2 l_{1} l_{2}}\right]\right)}{\sqrt{4 l_{1}^{2} l_{2}^{2}-\left(q_{r}^{2}-l_{1}^{2}-l_{2}^{2}\right)^{2}}} .
\end{aligned}
$$

To compare the Air Spring solution to those of the other springs, we numerically integrate the unperturbed stance dynamics from bottom $\left(p_{r}=0\right)$ to liftoff $\left(q_{r}=q_{r l}\right)$ for a large number of initial (bottom) conditions for both of these Hooke's law springs. Each of the simulated results is compared to the solutions given by (15) for the same initial conditions. For all the simulations shown in this paper we assume that $q_{r l}=1$ and $m=1$ and select the angle at bottom to be zero, $\theta_{b}=0$. The initial conditions are varied by changing the leg length, angular momentum, and spring potential energy at bottom. The data reported in Figure 3 arises from 1000 initial conditions ${ }^{7}$ selected from an equally

\footnotetext{
${ }^{7}$ All simulations are run from the same set of initial conditions. We take as the initial conditions $\theta_{b}=0, p_{r b}=0$ (ensuring a symmetric stance) and 1000 different values of $\left(r_{b}, p_{\theta b}, U\left(r_{b}\right)\right)$ selected from an equally spaced grid of the parameter cube, $r_{b} \in[0.75,0.975] \mathrm{m}, p_{\theta b} \in[1.5,6.5] \mathrm{kg} \mathrm{m} \mathrm{m}^{2} \mathrm{rad} / \mathrm{s}$, and $U\left(r_{b}\right) \in[0.25,6.25] \mathrm{kg} \mathrm{m}^{2} / \mathrm{s}^{2}$. This set of initial conditions results in a variety of gaits characterized by (for $U_{A}$, but similarly for $U_{H}$ and $\left.U_{T H K}\right) y_{a} \in[0.76,1.63] \mathrm{m}, \dot{x}_{a} \in[1.57,9.20] \mathrm{m} / \mathrm{s}$, and $\beta \in[0.02,0.45]$. For Figures 3 and 4 , which represent the unperturbed setting, only 982 of the 1000 initial conditions are summarized in this plot. Any initial conditions with low spring potential, large leg compression, and high angular velocity at bottom are ignored (simultaneously $U\left(r_{b}\right) \leq \frac{11}{12} \mathrm{~kg} \mathrm{~m} / \mathrm{s}^{2}, r_{b} \leq 0.80 \mathrm{~m}$, and $p_{\theta b} \geq \frac{29}{6} \mathrm{~kg} \mathrm{~m} \mathrm{~m}^{2} \mathrm{rad} / \mathrm{s}$ ) because they correspond to gaits which are "unrealistic" due to their simultaneously high velocities and high duty factors.
} 


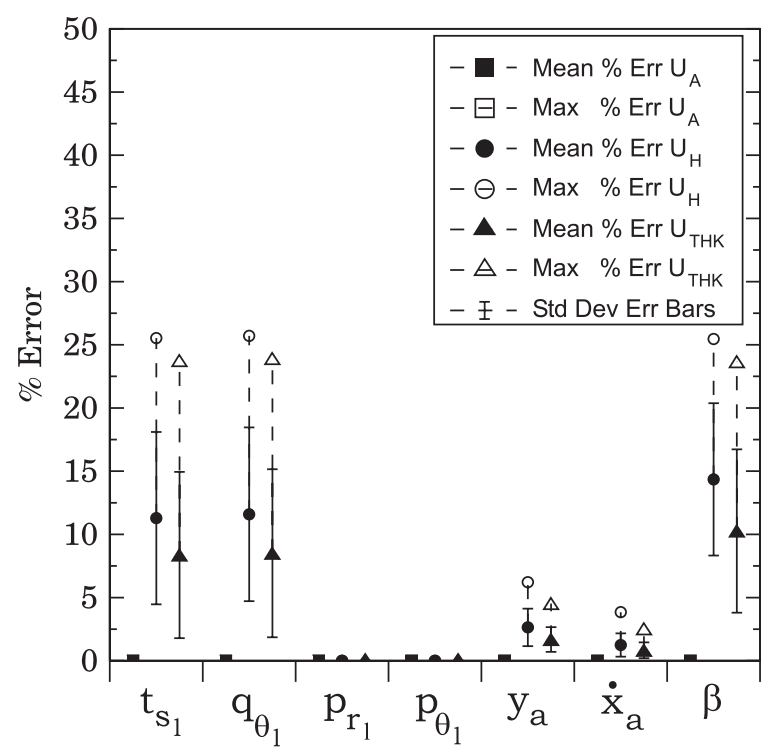

Fig. 3. Errors in liftoff and apex parameters arising from using the closed-form solution to the unperturbed stance dynamics for the Air Spring (15) as a functional approximant to the unperturbed dynamics (13) for the Air Spring, the Hooke's law spring, and a torsional Hooke's law spring at the knee of a revolute-revolute leg.

spaced grid within the cube formed by $r_{b} \in[0.75,0.975] \mathrm{m}, p_{\theta b} \in[1.5,6.5] \mathrm{kg} \mathrm{m} \mathrm{m}^{2} \mathrm{rad} / \mathrm{s}$, and $U\left(r_{b}\right) \in[0.25,6.25] \mathrm{kg} \mathrm{m} / \mathrm{s}^{2}$ with $\theta_{b}=0 \mathrm{rad}$. To make the comparisons as fair as possible, the spring constant for each respective Hooke's law spring is chosen to ensure that the spring potential energies at bottom are equal $\left(U_{A}\left(r_{b}\right)=U_{H}\left(r_{b}\right)=U_{T H K}\left(r_{b}\right)\right)$. As expected, there are no errors in $p_{r l}$ and $p_{\theta l}$, since we are considering the unperturbed case where both energy and angular momentum are constant. However, even so, the integrals for $t_{s}$ and $q_{\theta l}$ have maximum errors ${ }^{8}$ between $23.8 \%$ and $25.7 \%$ and mean errors between $8.22 \%$ and $11.4 \%$. Additionally, the apex variables $y_{a}$ and $\dot{x}_{a}$ have maximum errors between $2.53 \%$ and $6.22 \%$ and mean errors between $0.81 \%$ and $2.60 \%$, while the duty factor shows maximum errors between $23.7 \%$ and $25.5 \%$ and mean errors between $10.1 \%$ and $14.1 \%$.

Whether the errors ${ }^{9}$ reported in Figure 3 are acceptable or not may depend on the application of interest. For control applications, such as those discussed in Sec-

\footnotetext{
${ }^{8}$ We report errors in terms of percent errors (PE), where $P E=100 \frac{\| x \text { true }-x_{\text {approx }} \|_{2}}{\left\|x_{\text {true }}\right\|_{2}}$. See Appendix E for details.

${ }^{9}$ These results are for the unperturbed dynamics. The perturbed dynamics remain nonintegrable even for the Air Spring. Even so, simulation evidence suggests that using the "exact" solution for the Air Spring (obtained by numerical integration) as an approximant to that of the other springs in the perturbed case yields slightly worse results than those of Figure 3.
} 
tion 1.2, the addition of feedback correction may make the plant errors indicated in Figure 3 acceptable. However, for purposes of spring law identification, these errors may not be acceptable. Notice that in this case the errors in $y_{a}$ and $\dot{x}_{a}$ are reasonably small, but the duty factor has the highest mean errors. Since the comparison between the springs assumes a fixed leg length, angular momentum, and spring potential, these results suggest, loosely speaking, that the spring potential at bottom is a primary factor in determining hopping height, the angular momentum at bottom is a primary factor in determining forward speed, and the functional form of the spring is instrumental in determining duty factor. Therefore to more accurately identify spring laws in animal running, one would hope to have a better approximant for the duty factor.

\subsection{Approximate Solution for General Spring Law}

In the introduction we have tried to motivate the utility of physically parametrized closed-form approximations to the elliptic integrals (13), which arise from integrating the unperturbed SLIP dynamics with a general spring law, $U\left(q_{r}\right)$. For example, in control applications [32], the spring potential itself acts as a control input, and therefore it is important, if possible, to maintain the explicit appearance of the spring potential in the solutions.

The mean value theorem (MVT) for integrals states that under reasonable assumptions on $f(x)$ there exists a mean value, $\xi_{x}$, such that

$$
\int_{a}^{x} f(t) d t=f\left(\xi_{x}\right)(x-a) .
$$

In this manner, we can formally write down a closed-form integral solution for the unperturbed SLIP dynamics in which the spring law appears explicitly. Of course, the MVT only guarantees the existence of the mean value, $\xi_{x}$. It provides no means for computing it, and, in general, no exact method will be available. However, the strategy is attractive and we focus our attention on the utility of a linear approximation to the mean value function, $\xi_{x}$.

Specifically, in [38] we have shown

Theorem 1. Suppose $f$ is continuous on $(a, b]$ and $g$ is integrable on $(a, b)$ with $g(t) \geq 0$ for all $t \in(a, b)$. Let $x \in(a, b]$. If both

$$
\lim _{t \rightarrow a} \frac{f(t)-K}{(t-a)^{r}} \quad \lim _{t \rightarrow a} \frac{g(t)}{(t-a)^{s}}
$$

exist and are nonzero for some constant $K$, some nonzero $r$, and some $s>-1$ with $r+s>-1$, then

1. there exists $a \xi_{x} \in(a, x]$ such that

$$
\int_{a}^{x} f(t) g(t) d t=f\left(\xi_{x}\right) \int_{a}^{x} g(t) d t
$$


2. for any such choice of $\xi_{x}$,

$$
\lim _{x \rightarrow a} \frac{\xi_{x}-a}{x-a}=\left(\frac{s+1}{r+s+1}\right)^{\frac{1}{r}} .
$$

A practical observation [38] follows immediately from this theorem.

Observation 1. If, motivated by (20), we approximate $\xi_{x}$ by

$$
\hat{\xi}_{x}=a+\left(\frac{s+1}{r+s+1}\right)^{\frac{1}{r}}(x-a) \text { for } x \text { near } a,
$$

and replace $\xi_{x}$ by $\hat{\xi}_{x}$ in (19), we obtain an approximation scheme to the integral

$$
\int_{a}^{x} f(t) g(t) d t \approx f\left(\hat{\xi}_{x}\right) \int_{a}^{x} g(t) d t \quad \text { for } x \text { near } a .
$$

For the case at hand, applying Theorem 1 and Observation 1, we find

$$
\begin{aligned}
& p_{\theta}\left(q_{r}\right)=p_{\theta b}, \\
& p_{r}\left(q_{r}\right)=\left[p_{\theta b}^{2}\left(\frac{1}{r_{b}^{2}}-\frac{1}{q_{r}^{2}}\right)+2 m\left(U\left(r_{b}\right)-U\left(q_{r}\right)\right)\right]^{\frac{1}{2}}, \\
& \hat{q}_{\theta}\left(q_{r}\right)=\frac{p_{\theta b}\left(q_{r}-r_{b}\right)}{\hat{\xi}_{r}^{2}\left[p_{\theta b}^{2}\left(\frac{1}{r_{b}^{2}}-\frac{1}{\hat{\xi}_{r}^{2}}\right)+2 m\left(U\left(r_{b}\right)-U\left(\hat{\xi}_{r}\right)\right)\right]^{\frac{1}{2}}}, \\
& \hat{t}_{s}\left(q_{r}\right)=\frac{m\left(q_{r}-r_{b}\right)}{\left[p_{\theta b}^{2}\left(\frac{1}{r_{b}^{2}}-\frac{1}{\hat{\xi}_{r}^{2}}\right)+2 m\left(U\left(r_{b}\right)-U\left(\hat{\xi}_{r}\right)\right)\right]^{\frac{1}{2}}},
\end{aligned}
$$

where under very reasonable assumptions on the spring potential (see Appendix A) we have

$$
\hat{\xi}_{r}=r_{b}+\frac{1}{4}\left(q_{r}-r_{b}\right) \text {. }
$$

Beyond yielding a good approximation when $q_{r}$ is close to $r_{b}$, these approximations are quite effective over a reasonably large portion of the parameter space. Figure 4 summarizes simulation data ${ }^{7}$ for the $U_{A}, U_{H}$, and $U_{T H K}$ springs. In each case the maximum percent error is less than $12 \%$ and the mean percent error is less than $2.7 \%$. Comparing with Figure 3, one sees that this Mean Value approach results in better approximants to the Hooke's Law cases $\left(U_{H}, U_{T H K}\right)$ than using the Air Spring solutions (15). In particular, the maximum errors in $t_{s}$ and $q_{\theta l}$ drop from more than $23 \%$ to less than $3.75 \%$, while the mean errors drop from more than $8.3 \%$ to less than $2.7 \%$. Also the maximum errors in duty factor, $\beta$, drop from more than $23 \%$ to less than $12 \%$ and the mean errors from more than $10 \%$ to less than $2 \%$.

\section{4. "Integration" of the Perturbed Stance Dynamics}

In the previous section, we "turned off" gravity during stance, introduced a good approximation method for the elliptic integrals, and exhibited a closed-form bottom-to-apex 


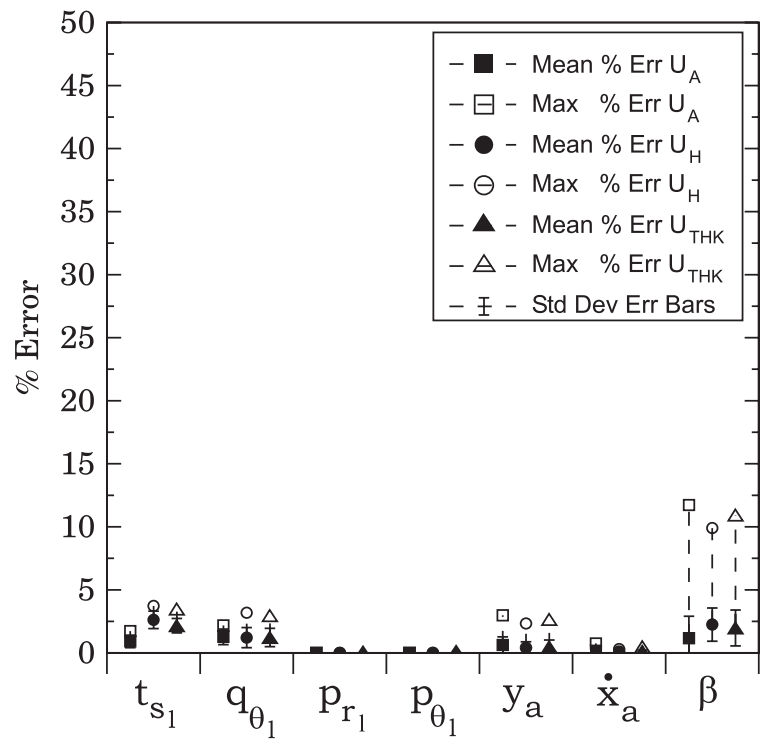

Fig. 4. Errors in liftoff and apex parameters arising from using mean-value inspired approximations of (23) as a functional approximant to the unperturbed dynamics (13) for the Air Spring, the Hooke's Law Spring, and a Torsional Hooke's Law Spring at the knee of a revolute-revolute leg.

map. Our choice to ignore gravity during stance is not uncommon in the robotics literature [22], [27], [40], [43]. In these papers, this choice is made primarily to simplify the analysis and relies upon the assumptions that the spring force dominates the gravitational force and that the angular momentum is nearly constant during stance. Under such assumptions the unperturbed mapping derived in the last section should work well.

In order to test how well, we repeat the simulations of Figure 4, except in this case we compare the results of the unperturbed MVT approximants (23) and (24) to the simulation results of the perturbed system. The data, reported in Figure 5, arises from 1000 initial conditions ${ }^{10}$ selected from an equally spaced grid within the cube formed by $r_{b} \in[0.75,0.975] \mathrm{m}, p_{\theta b} \in[1.5,6.5] \mathrm{kg} \mathrm{m}^{2} \mathrm{rad} / \mathrm{s}$, and $U\left(r_{b}\right) \in[2.5,7.25] \mathrm{kg} \mathrm{m}^{2} / \mathrm{s}^{2}$ with $\theta_{b}=0 \mathrm{rad}$.

In this case the maximum errors are as high as $60 \%$ and the mean errors are as high as $20 \%$. These are considerably worse than the results shown in Figure 4, demonstrating that the effects of gravity cannot be ignored in general locomotion. In other words, the general assumptions made to justify ignoring gravity in stance are not valid over a large range of running gaits.

\footnotetext{
${ }^{10}$ All simulations are run from the same set of initial conditions. We take as the initial conditions $\theta_{b}=0$, $p_{r b}=0$ (ensuring a symmetric stance), and 1000 different values of $\left(r_{b}, p_{\theta b}, U\left(r_{b}\right)\right)$ selected from an equally spaced grid of the parameter cube, $r_{b} \in[0.75,0.975], p_{\theta b} \in[1.5,6.5]$, and $U\left(r_{b}\right) \in[2.5,7.5]$. These initial conditions produce a variety of gaits characterized by (for $U_{A}$, but similarly for $U_{H}$ and $U_{T H K}$ ) $y_{a} \in[0.76,1.73] \mathrm{m}, \dot{x}_{a} \in[1.58,9.34] \mathrm{m} / \mathrm{s}$, and $\beta \in[0.02,0.46]$.
} 


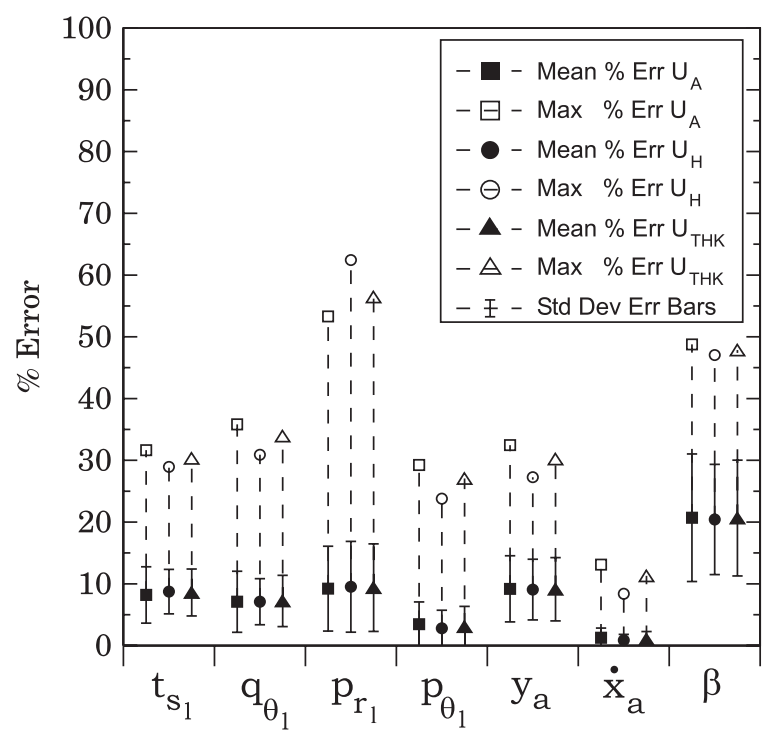

Fig. 5. Errors in liftoff and apex parameters in the approximate mappings of the unperturbed system (23) when compared to the "true" simulated values of the perturbed system. Errors are computed for three springs introduced in Section 3.2, i.e. the compressed air spring, $U_{A}\left(q_{r}\right)$, the Hooke's Law Spring, $U_{H}\left(q_{r}\right)$, and the effective spring arising from a Torsional Hooke's Law Spring at the knee, $U_{T H K}\left(q_{r}\right)$.

This motivates the need for a reasoned approach to modify the unperturbed mapping to account for the presence of gravity.

\section{1. "Exact” Gravity Correction}

The presence of the gravitational perturbation preserves total energy as a constant of motion, but not angular momentum. Therefore the perturbed dynamics can be rewritten as the following set of three coupled nonlinear differential equations,

$$
\begin{aligned}
& \frac{d t_{s}}{d q_{r}}\left(q_{r}, q_{\theta}, p_{\theta}\right)=\frac{m}{p_{r}\left(q_{r}, q_{\theta}, p_{\theta}\right)}, \\
& \frac{d q_{\theta}}{d q_{r}}\left(q_{r}, q_{\theta}, p_{\theta}\right)=\frac{p_{\theta}}{q_{r}^{2} p_{r}\left(q_{r}, q_{\theta}, p_{\theta}\right)}, \\
& \frac{d p_{\theta}}{d q_{r}}\left(q_{r}, q_{\theta}, p_{\theta}\right)=\frac{m^{2} g q_{r} \sin \left(q_{\theta}\right)}{p_{r}\left(q_{r}, q_{\theta}, p_{\theta}\right)},
\end{aligned}
$$

with the additional constraint imposed by constant energy: $p_{r}$ solves the implicit function $H\left(q_{r}, q_{\theta}, p_{r}, p_{\theta}\right)=H_{0}$ that we denote by

$$
p_{r}\left(q_{r}, q_{\theta}, p_{\theta}, H_{0}\right)=H^{-1}\left(q_{r}, q_{\theta}, p_{\theta}, H_{0}\right)
$$


For ease of exposition, introduce the following notation: $y=\left[t_{s}, q_{\theta}, p_{\theta}\right]^{T}$ and $z=$ $H^{-1}\left(q_{r}, y\right)$. Notice that the explicit dependence of $H^{-1}$ on $H_{0}$ has been dropped for simplicity. Now, we can express the system of (25) and (26) as

$$
\begin{aligned}
y^{\prime} & =f_{p}\left(q_{r}, y, z\right), \\
z & =H^{-1}\left(q_{r}, y\right) .
\end{aligned}
$$

\subsection{Integration of the Perturbed System Using Unperturbed Solutions}

As observed earlier, this system cannot be solved in general. However, we do have a solution to the unperturbed system given in (13). Let these solutions be denoted by $y_{0}\left(q_{r}\right)$ and $z_{0}\left(q_{r}\right)=H^{-1}\left(q_{r}, y_{0}\right)$.

Since these unperturbed solutions are functions solely of $q_{r}$, our strategy is to substitute these approximations into the right-hand side of (27) for $y$ and $z$. The result is a set of separable differential equations which can easily be written as integrals in terms of $q_{r}$. This observation motivates the following iteration procedure, ${ }^{11}$ where the unperturbed solution is used to generate iterates that converge to the solution of the perturbed system,

$$
\begin{aligned}
& y_{(n+1)}\left(q_{r}\right)=y_{b}+\int_{r_{b}}^{q_{r}} f_{p}\left(\rho, y_{n}(\rho), H^{-1}\left(\rho, y_{n}(\rho)\right)\right) d \rho, \\
& z_{(n+1)}\left(q_{r}\right)=H^{-1}\left(q_{r}, y_{(n+1)}\left(q_{r}\right)\right) .
\end{aligned}
$$

\subsection{Approximate Integration of the Perturbed System: An Iterative Approach}

As in the unperturbed case, we can approximate the integrals of interest using Theorem 1 and Observation 1, obtaining $\hat{y}_{0}\left(q_{r}\right)$ and $\hat{z}_{0}\left(q_{r}\right)=H^{-1}\left(q_{r}, \hat{y}_{0}\right)$ as shown in (23).

Using these solutions and again applying the MVT result of Section 3.3, we can write the iteration as

$$
\begin{aligned}
& \hat{y}_{(n+1)}\left(q_{r}\right)=y_{b}+f_{p}\left(\hat{\xi}_{r}, \hat{y}_{n}\left(\hat{\xi}_{r}\right), H^{-1}\left(\hat{\xi}_{r}, \hat{y}_{n}\left(\hat{\xi}_{r}\right)\right)\right)\left(q_{r}-r_{b}\right), \\
& \hat{z}_{(n+1)}\left(q_{r}\right)=H^{-1}\left(q_{r}, \hat{y}_{(n+1)}\left(q_{r}\right)\right) .
\end{aligned}
$$

Expressed in all its detail, the procedure appears as

$$
\begin{aligned}
& \hat{t}_{s(n+1)}\left(q_{r}\right)=t_{s b}+\frac{m}{H_{p e r t}^{-1}\left(\hat{\xi}_{r}, \hat{q}_{\theta n}\left(\hat{\xi}_{r}\right), \hat{p}_{\theta n}\left(\hat{\xi}_{r}\right)\right)}\left(q_{r}-r_{b}\right), \\
& \hat{q}_{\theta(n+1)}\left(q_{r}\right)=\theta_{b}+\frac{\hat{p}_{\theta n}\left(\hat{\xi}_{r}\right)}{\hat{\xi}_{r}^{2} H_{p e r t}^{-1}\left(\hat{\xi}_{r}, \hat{q}_{\theta n}\left(\hat{\xi}_{r}\right), \hat{p}_{\theta_{n}}\left(\hat{\xi}_{r}\right)\right)}\left(q_{r}-r_{b}\right), \\
& \hat{p}_{\theta(n+1)}\left(q_{r}\right)=p_{\theta b}+\frac{m^{2} g \hat{\xi}_{r} \sin \left(\hat{q}_{\theta n}\left(\hat{\xi}_{r}\right)\right)}{H_{p e r t}^{-1}\left(\hat{\xi}_{r}, \hat{q}_{\theta n}\left(\hat{\xi}_{r}\right), \hat{p}_{\theta_{n}}\left(\hat{\xi}_{r}\right)\right)}\left(q_{r}-r_{b}\right),
\end{aligned}
$$

\footnotetext{
${ }^{11}$ Note that the previous iterate is used to construct $y_{(n+1)}$, but the current iterate is used to construct $z_{(n+1)}$. This choice is made because it preserves constant energy for all iterates. See Appendix D for the details.
} 


$$
\begin{aligned}
\hat{p}_{r(n+1)}\left(q_{r}\right)= & {\left[2 m\left[\left(U\left(r_{b}\right)-U\left(q_{r}\right)\right)+m g\left(r_{b} \cos \left(\theta_{b}\right)-q_{r} \cos \left(\hat{q}_{\theta(n+1)}\left(q_{r}\right)\right)\right)\right]\right.} \\
& \left.+\left(\frac{p_{\theta b}^{2}}{r_{b}^{2}}-\frac{\hat{p}_{\theta(n+1)}^{2}\left(q_{r}\right)}{q_{r}^{2}}\right)\right]^{\frac{1}{2}},
\end{aligned}
$$

where in each case we find $\hat{\xi}_{r}=r_{b}+\frac{1}{4}\left(q_{r}-r_{b}\right)$ (see Appendix A).

This iteration method for generating the perturbed solution is strongly reminiscent of Picard iterations, which are used to prove the existence and uniqueness of solutions of differential equations. This relation to Picard iterates suggests that the iteration procedure of (31) and (32) would converge to the correct solution if the exact mean value were known. Recall that the mean value approximations introduced in Section 3.3 are "exact" as $q_{r}$ approaches $r_{b}$. It can be shown (see Appendix C) that under appropriate assumptions the combination of the iteration steps and MVT approximations converges to the solution of the perturbed dynamics for $q_{r}$ close to $r_{b}$.

There remains one difficulty. The result presented in Appendix $\mathrm{C}$ assumes the vector field is continuous and Lipschitz. While the original Hamiltonian system meets all required continuity and Lipschitz conditions, the "quadrature" system, (25) and (26), set up to integrate this Hamiltonian system does not $-p_{r}$ is in the denominator of equations (25) and, by definition, $p_{r}=0$ at the bottom point (the selected initial condition). Therefore, these equations are neither continuous nor Lipschitz on a closed region containing the initial conditions of interest. However, $1 / p_{r}$ "blows up" in a particularly nice manner as $q_{r}$ goes to $r_{b}$, that is, in an integrable fashion (as $\left.1 /\left(q_{r}-r_{b}\right)^{\frac{1}{2}}\right)$. In fact, it is exactly this property that allows the application of Theorem 1 to this problem. This suggests that a similar proof may exist for the existence and uniqueness properties of this system. We are currently pursuing such an argument.

\subsection{Rate of Convergence Studies}

From the perspective of engineering applications, we are concerned with finding a "good" approximate plant model. In other words, we would like to know how many iterations it takes to get "close enough" to the actual solution over a sufficiently large region of interest. ${ }^{12}$ In traditional Picard iterations, it is possible to place a bound on the error between the solution and a particular iterate. However, in our case, because of the errors introduced by violating the $q_{r}$ close to $r_{b}$ assumption, we resort to simulation evidence as in Figure 6. In these simulations, the zeroth approximant is given by the approximate solutions to the unperturbed system (23), while first and second approximants are generated using the iteration procedure of (33). In both cases the mean value is given by (24). These results are compared to simulation results of the perturbed system for the same set of initial conditions ${ }^{10}$ considered in Figure 5. Notice that the plots show a progression where both the maximum and mean errors are reduced for each increasing

\footnotetext{
12 If the iteration scheme presented in (31) and (32) does not perform sufficiently for a particular application, two alternative approximate solutions for the perturbed SLIP are presented in Appendix F.
} 

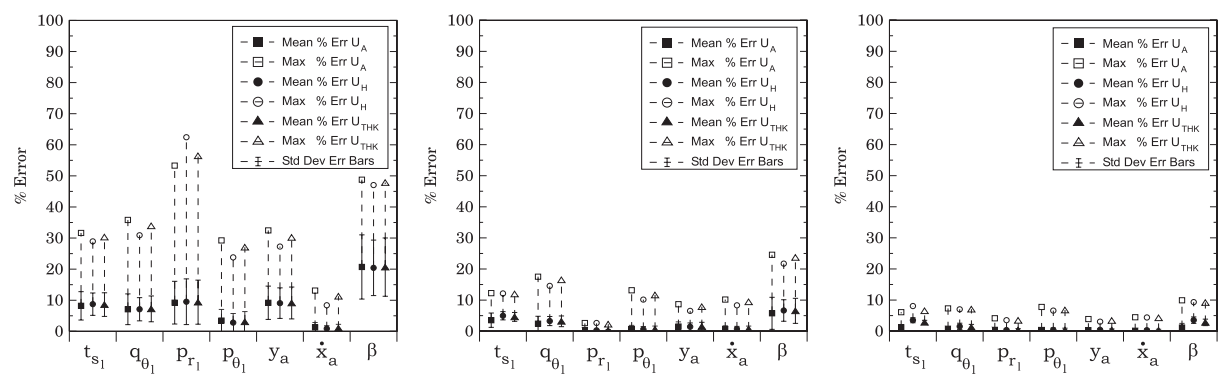

Fig. 6. Errors in liftoff and apex parameters in the approximate iterates of (33) when compared to the "true" simulated values of the perturbed system: (left) zeroth iterate (unperturbed solution); (middle) first iterate; (right) second iterate. Errors are computed for three springs introduced in Section 3.2, i.e. the compressed air spring, $U_{A}\left(q_{r}\right)$, the Hooke's Law Spring, $U_{H}\left(q_{r}\right)$, and the effective spring arising from a Torsional Hooke's Law Spring at the knee, $U_{T H K}\left(q_{r}\right)$.

iterate. The largest maximum errors proceed from $60 \%$ for the zeroth iterate to $25 \%$ for the first iterate to $10 \%$ for the second iterate. Similarly the largest mean errors decrease from $20 \%$ to $7 \%$ to $3.5 \%$ as the iterates increase.

The goal of this research is to develop plant models valid over a large range of running and hopping gaits. For this reason, it is important to test the approximation for a sufficiently large set of bottom initial conditions. Of course, what constitutes such a set is somewhat difficult to determine, since the meaning of the bottom coordinates is not easily interpreted in our experience. The apex coordinate system, which is specified in terms of height, velocity, and duty factor, is a much more natural set of coordinates for interpreting gaits. Figure 7(a) is a scatter plot of the 1000 initial bottom conditions used to generate the data of Figure 6. The resulting set of apex coordinates generated from this set of bottom initial coordinates is shown in the scatter plot of Figure 7(b). It is seen that the initial bottom conditions provide a rich set of apex gaits which, when interpreted in terms of a human's ability, include hopping heights which range from barely getting off the ground ${ }^{13}$ to Michael Jordan-like jumping, $y_{a} \in[0.77,1.74] \mathrm{m}$; forward velocities which include the the slowest runner as well as those with near world-class speed, $\dot{x}_{a} \in[1.56,9.26] \mathrm{m} / \mathrm{s}$; and duty factors which range from almost never on the ground to almost always on the ground, $\beta \in[0.01,0.41]$.

\section{Conclusion}

Motivated by the need for a "plant model" for robot and animal running, we propose a relatively simple means of approximating the integral maps of a class of nonintegrable Hamiltonian systems. By appeal to an appropriate generalization of the mean value theorem [38], the approximants involve closed-form expressions read directly from the

\footnotetext{
13 Apex heights less than the nominal leg length, $1 \mathrm{~m}$ in these simulations, would require the leg to be retracted in order to swing into position for the next touchdown.
} 

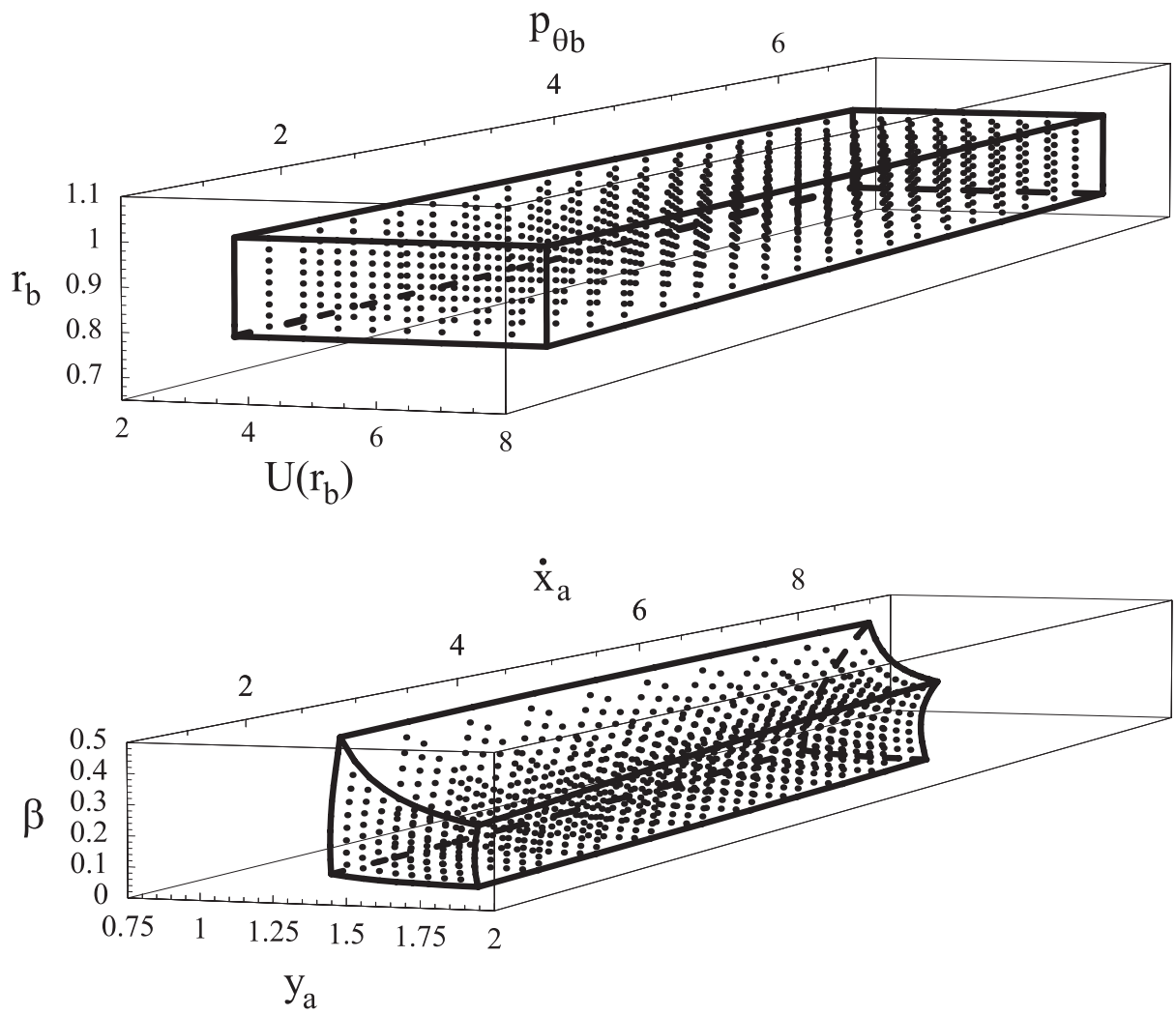

Fig. 7. (a) Top: Scatter Plot of the set of bottom initial conditions used to calculate the errors of the approximation procedures presented in this section. Shown are 1000 different initial conditions $\left(r_{b}, p_{\theta b}, U\left(r_{b}\right)\right)$ selected from an equally spaced grid of the parameter cube, $r_{b} \in[0.75,0.975] \mathrm{m}$, $p_{\theta b} \in[1.5,6.5] \mathrm{kg} \mathrm{m}^{2} \mathrm{rad} / \mathrm{s}$, and $U\left(r_{b}\right) \in[2.5,7.5] \mathrm{kg} \mathrm{m}^{2} / \mathrm{s}^{2}$. (b) Bottom: Scatter Plot of the set of apex final conditions arising from integrating the set of bottom initial conditions through the SLIP dynamics with a Hooke's law spring. The results are gaits which include hopping heights in the range $y_{a} \in[0.77,1.74] \mathrm{m}$, forward velocities in the range $\dot{x}_{a} \in[1.56,9.26] \mathrm{m} / \mathrm{s}$, and duty factors in the range $\beta \in[0.01,0.41]$. Simulations were run assuming a leg length of $1 \mathrm{~m}$ and a mass of $1 \mathrm{~kg}$.

Hamiltonian vector field. By casting the method as an iterative procedure, we gain reasonable control over the inevitable tradeoff between accuracy and simplicity.

Although inspired by our specific interest in the stance map arising from a simple model of legged locomotion, the procedure can be applied to any Hamiltonian system expressed as a sum of an integrable along with a (not necessarily small) perturbation term. Thus we have some reason to hope that the method reported here may generalize to a larger class of problems. Because the approximants involve exact linearization of the mean value function at one end point of the integral, their accuracy falls off as the desired interval of integration increases. However, we have found them quite useful in this running work because human-sized bipeds have a single-leg stance phase which is quite short. Conceivably, in applications where longer intervals of integration are critical, 
generalizations of this idea involving either higher order estimates of the mean value function or subdivisions of the integration range into shorter constituent intervals may work effectively.

\section{Appendix A. Derivation of the Mean Value Function Approximant, $\hat{\xi}_{x}$}

Begin by considering the $t_{s}\left(q_{r}\right)$ integral for the perturbed case. In (19) choose

$$
g_{t s}\left(q_{r}\right)=1
$$

and

$$
f_{t s}\left(q_{r}\right)=\frac{m}{\left[\left(\frac{p_{\theta b}^{2}}{r_{b}^{2}}-\frac{p_{\theta}^{2}}{q_{r}^{2}}\right)+2 m\left(\left(U\left(r_{b}\right)-U\left(q_{r}\right)\right)+m g\left(r_{b} \cos \left(\theta_{b}\right)-q_{r} \cos \left(q_{\theta}\right)\right)\right)\right]^{\frac{1}{2}}} .
$$

In this case $s_{t_{s}}=0$. Choose $K=0$. Then by Theorem 1 we seek $r_{t_{s}}$ such that the following limit exists and is nonzero:

$$
\lim _{q_{r} \rightarrow r_{b}} \frac{f_{t s}\left(q_{r}\right)}{\left(q_{r}-r_{b}\right)^{r_{t_{s}}}}=\lim _{q_{r} \rightarrow r_{b}} \frac{m\left(q_{r}-r_{b}\right)^{\left(-r_{t_{s}}-\frac{1}{2}\right)}}{\left[\frac{\left[\left(\frac{p_{\theta b}^{2}}{r_{b}^{2}}-\frac{p_{\theta}^{2}}{q_{r}^{2}}\right)+2 m\left(\left(U\left(r_{b}\right)-U\left(q_{r}\right)\right)+m g\left(r_{b} \cos \left(\theta_{b}\right)-q_{r} \cos \left(q_{\theta}\right)\right)\right)\right.}{\left(q_{r}-r_{b}\right)}\right]^{\frac{1}{2}}} .
$$

Select $r_{t_{s}}=-\frac{1}{2}$. The numerator of (34) simplifies to $m$, and the question reduces to whether the following limit exists and is nonzero,

$$
\lim _{q_{r} \rightarrow r_{b}} \frac{\left[\left(\frac{p_{\theta b}^{2}}{r_{b}^{2}}-\frac{p_{\theta}^{2}}{q_{r}^{2}}\right)+2 m\left(\left(U\left(r_{b}\right)-U\left(q_{r}\right)\right)+m g\left(r_{b} \cos \left(\theta_{b}\right)-q_{r} \cos \left(q_{\theta}\right)\right)\right)\right]}{\left(q_{r}-r_{b}\right)} .
$$

Straightforward evaluation of this limit gives $\frac{0}{0}$. Applying L'Hospital's rule and substituting the results of equation (25), we find

$$
\begin{aligned}
& \lim _{q_{r} \rightarrow r_{b}} \frac{\left[\left(\frac{p_{\theta b}^{2}}{r_{b}^{2}}-\frac{p_{\theta}^{2}}{q_{r}^{2}}\right)+2 m\left(\left(U\left(r_{b}\right)-U\left(q_{r}\right)\right)+m g\left(r_{b} \cos \left(\theta_{b}\right)-q_{r} \cos \left(q_{\theta}\right)\right)\right)\right]}{\left(q_{r}-r_{b}\right)} \\
& =\lim _{q_{r} \rightarrow r_{b}}\left[\frac{2 p_{\theta}^{2}}{q_{r}^{3}}-\frac{2 p_{\theta} p_{\theta}^{\prime}}{q_{r}^{2}}-2 m D U\left(q_{r}\right)+2 m^{2} g\left(q_{r} q_{\theta}^{\prime} \sin \left(q_{\theta}\right)-\cos \left(q_{\theta}\right)\right)\right] \\
& =\lim _{q_{r} \rightarrow r_{b}}\left[\frac{2 p_{\theta}^{2}}{q_{r}^{3}}-\frac{2 p_{\theta} m^{2} g \sin \left(q_{\theta}\right)}{q_{r} p_{r}}-2 m D U\left(q_{r}\right)+2 m^{2} g\left(\frac{p_{\theta}}{q_{r} p_{r}} \sin \left(q_{\theta}\right)-\cos \left(q_{\theta}\right)\right)\right] \\
& =\lim _{q_{r} \rightarrow r_{b}}\left[\frac{2 p_{\theta}^{2}}{q_{r}^{3}}-2 m D U\left(q_{r}\right)-2 m^{2} g \cos \left(q_{\theta}\right)\right] .
\end{aligned}
$$

This limit exists and is nonzero under very reasonable assumptions on $U\left(q_{r}\right)$, namely that $D U\left(r_{b}\right)$ exists and

$$
D U\left(r_{b}\right) \neq \frac{p_{\theta b}^{2}}{m r_{b}^{3}}-m g \cos \theta_{b}
$$


In this case, substituting $r_{t s}$ and $s_{t_{s}}$ into (21) yields (24). The result is shown similarly for $q_{\theta}\left(q_{r}\right)$ and $p_{\theta}\left(q_{r}\right)$.

Note from (4) and (5) that the exception $D U\left(r_{b}\right)=\frac{p_{\theta b}^{2}}{m r_{b}^{3}}-m g \cos \theta_{b}$ implies $\dot{p}_{r}\left(r_{b}\right)=$ 0 . Recall also that $r_{b}$ is defined such that $p_{r}\left(r_{b}\right)=0$. For the unperturbed case $(g=0)$ in which angular momentum is constant, it can be shown that this condition implies $\dot{q}_{r}\left(q_{r}\right)=0$. In other words, for the unperturbed case, the physical interpretation of this exception is that the SLIP is acting as a stiff pendulum. That is, the leg is spinning about the "toe" at a constant angular velocity with no change in leg length.

The physical interpretation is not so clear in the perturbed case. However, one can easily see that the set of bottom conditions for which (37) is violated will be a "thin" set and therefore unlikely to occur.

\section{Appendix B. SLSK Leg}

While bearing strong resemblance to the physical construction of many running robots [32], [15], the SLIP leg bears little resemblance to animal legs, since they generally have revolute, not prismatic, joints.

To begin to make the connection to more biologically plausible models, we introduce the simplest physical correlate, the revolute-revolute leg with a spring at the knee, shown in Figure 8. This leg will be referred to as the SLSK (Spring-Loaded Small Knee) leg. In the case of negligible leg (knee) mass, there is a change of coordinates-an isometry, in fact-between the SLIP and SLSK leg motions. ${ }^{14}$

As Figure 8 suggests, for any SLSK spring, there is a well-defined SLIP spring and vice versa (the two are related, of course, through the transposed jacobian of the isometry), such that SLSK motion from any initial condition can be read off the motion of the SLIP mapped back through the isometry (assuming appropriately chosen SLIP initial conditions). Since all properties of interest are invariant under change of coordinates, and since the isometry can be written in closed form, it follows that we can derive the same insights from either leg model.

\section{B.1. SLSK/SLIP Isometry}

Because each of the coordinate systems (SLSK, SLIP) may be useful, we introduce the following maps between them, $\boldsymbol{b}=g(\boldsymbol{q})=\bar{g}(\overline{\boldsymbol{q}})$, where

$$
g(\boldsymbol{q})=\left[\begin{array}{c}
q_{r} \sin q_{\theta} \\
q_{r} \cos q_{\theta}
\end{array}\right]
$$

and

$$
\bar{g}(\overline{\boldsymbol{q}})=\left[\begin{array}{c}
l_{1} \cos \bar{q}_{\theta_{1}}+l_{2} \cos \left(\bar{q}_{\theta_{1}}+\bar{q}_{\theta_{2}}\right) \\
l_{1} \sin \bar{q}_{\theta_{1}}+l_{2} \sin \left(\bar{q}_{\theta_{1}}+\bar{q}_{\theta_{2}}\right)
\end{array}\right] .
$$

\footnotetext{
${ }^{14}$ To be exact, the isometry breaks at the critical points (straight leg and doubled over leg), reflecting the fact that the torus is a double cover of the punctured disk—see Appendix B.1 for details. It should be intuitively clear, however, that monoped legs will not operate anywhere near these critical points.
} 


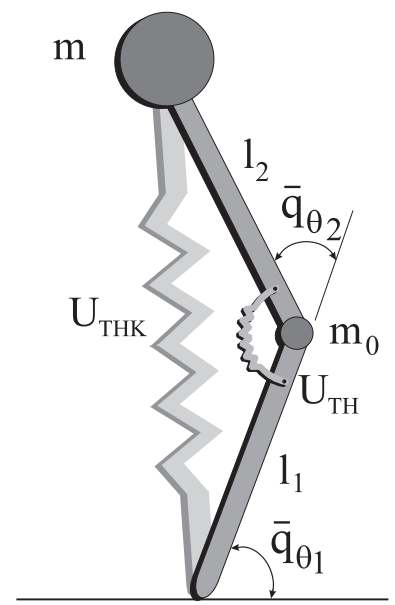

Fig. 8. The spring-loaded inverted pendulum (SLIP) monoped is dynamically equivalent to the SLSK monoped when there is no mass as the knee $\left(m_{0}=0\right)$.

Let the pair $\left(\mathcal{X}, M_{\mathcal{X}}\right)$ represent the space $\mathcal{X}$ endowed with the metric, $M_{\mathcal{X}}$, which defines a norm (the kinetic energy) on the tangent space at each point, $T \mathcal{X}_{p}$. We will represent $M_{\mathcal{X}}$ as a matrix, but it should be understood that for $x_{p} \in \mathcal{X}$, the metric is applied to the tangent vector, $\dot{x}_{p} \in T \mathcal{X}_{p}$, in the following manner, $\dot{x}_{p}^{T} M_{\mathcal{X}} \dot{x}_{p} / 2$.

In this work, we will find it advantageous to consider the metric spaces, $\left(\mathcal{B}, M_{\mathcal{B}}\right)$, $\left(\mathcal{Q}, M_{\mathcal{Q}}\right)$, and $\left(\overline{\mathcal{Q}}, M_{\overline{\mathcal{Q}}}\right)$, where

and

$$
\begin{aligned}
M_{\mathcal{B}} & =m\left[\begin{array}{ll}
1 & 0 \\
0 & 1
\end{array}\right], \\
M_{\mathcal{Q}} & =m D_{\boldsymbol{q}} g^{T} D_{\boldsymbol{q}} g \\
& =m\left[\begin{array}{cc}
1 & 0 \\
0 & q_{r}^{2}
\end{array}\right],
\end{aligned}
$$

$$
\begin{aligned}
M_{\overline{\mathcal{Q}}} & =m D \overline{\boldsymbol{q}}^{T} \bar{D}_{\overline{\boldsymbol{q}}} \overline{\mathrm{g}} \\
& =m\left[\begin{array}{cc}
l_{1}^{2}+l_{2}^{2}+2 l_{1} l_{2} \cos \bar{q}_{\theta_{2}} & l_{2}^{2}+l_{1} l_{2} \cos \bar{q}_{\theta_{2}} \\
l_{2}^{2}+l_{1} l_{2} \cos \bar{q}_{\theta_{2}} & l_{2}^{2}
\end{array}\right] .
\end{aligned}
$$

By construction the maps $g:\left(\mathcal{Q}, M_{\mathcal{Q}}\right) \mapsto\left(\mathcal{B}, M_{\mathcal{B}}\right)$ and $\bar{g}:\left(\overline{\mathcal{Q}}, M_{\overline{\mathcal{Q}}}\right) \mapsto\left(\mathcal{B}, M_{\mathcal{B}}\right)$ are isometries. Therefore the map $g^{-1} \circ \bar{g}:\left(\overline{\mathcal{Q}}, M_{\overline{\mathcal{Q}}}\right) \mapsto\left(\mathcal{Q}, M_{\mathcal{Q}}\right)$,

$$
h:=g^{-1} \circ \bar{g}=\left[\begin{array}{c}
\sqrt{l_{1}^{2}+l_{2}^{2}+2 l_{1} l_{2} \cos \bar{q}_{\theta_{2}}} \\
-\bar{q}_{\theta_{1}}+\arccos \left[\frac{l_{2} \sin \bar{q}_{\theta_{2}}}{\sqrt{l_{1}^{2}+l_{2}^{2}+2 l_{1} l_{2} \cos \bar{q}_{\theta_{2}}}}\right]
\end{array}\right],
$$


is an isometry.

In the case that all the mass is concentrated at the hip $\left(m_{0}=0\right)$, the metrics introduced above are exactly the kinetic energy. In this case, the Lagrangian vector field corresponding to the SLSK dynamics, $L_{\bar{q}}$, can then be given in terms of the Lagrangian vector field corresponding to the SLIP dynamics, $L_{q}$, and the change of coordinates, $h$, as

$$
L_{\bar{q}}=D\left(T h^{-1}\right) \circ L_{q} \circ T h,
$$

where $T h$ denotes the tangent map of $h$.

\section{B.2. Torsional Spring at the Knee of SLSK Leg}

Consider a Torsional Hooke's Law Spring in the knee of the SLSK model given by $U_{T H}\left(\bar{q}_{\theta_{2}}\right)=\frac{k}{2}\left(\bar{q}_{\theta_{2}}-\bar{q}_{\theta_{2} l}\right)^{2}$. Consequently,

$$
D U_{T H}\left(\bar{q}_{\theta_{2}}\right)=k\left(\bar{q}_{\theta_{2}}-\bar{q}_{\theta_{2} l}\right),
$$

where

$$
\bar{q}_{\theta_{2} l}=\arccos \left[\frac{q_{r l}^{2}-l_{1}^{2}-l_{2}^{2}}{2 l_{1} l_{2}}\right] .
$$

The equivalent SLIP spring law is given by

$$
\begin{aligned}
D U_{T H K} & =[1,0] D \boldsymbol{q} h^{-1 T}\left[0, D U_{T H} \circ h^{-1}\right]^{T} \\
& =-2 q_{r} k \frac{\left(\arccos \left[\frac{q_{r}^{2}-l_{1}^{2}-l_{2}^{2}}{2 l_{1} l_{2}}\right]-\arccos \left[\frac{q_{r l}^{2}-l_{1}^{2}-l_{2}^{2}}{2 l_{1} l_{2}}\right]\right)}{\sqrt{4 l_{1}^{2} l_{2}^{2}-\left(q_{r}^{2}-l_{1}^{2}-l_{2}^{2}\right)^{2}}} .
\end{aligned}
$$

The spring potential in this case is given by

$$
\begin{aligned}
U_{T H K} & =U_{T H} \circ h^{-1} \\
& =\frac{k}{2}\left(\arccos \left[\frac{q_{r}^{2}-l_{1}^{2}-l_{2}^{2}}{2 l_{1} l_{2}}\right]-\arccos \left[\frac{q_{r l}^{2}-l_{1}^{2}-l_{2}^{2}}{2 l_{1} l_{2}}\right]\right)^{2} .
\end{aligned}
$$

\section{Appendix C. Picard Iterations}

\section{C.1. Existence and Uniqueness Theorems}

For studying the existence and uniqueness of differential equations, one of the simplest results is the Picard-Lindelöf Theorem [18], [41] shown below. There are, of course, other theorems that can be used, but Picard-Lindelöf has the additional advantage of providing in its proof a method for constructing a solution to the equations - the successive Picard iterations.

Theorem 2. Let $f(x, y)$ be continuous and Lipschitz on $R: x_{0} \leq x \leq x_{0}+a,\left|y-y_{0}\right| \leq b$. There exists a number $h>0$ with the property that the initial value problem

$$
y^{\prime}=f(x, y) \quad y\left(x_{0}\right)=y_{0}
$$


has one and only one solution, $y=y(x)$, on the interval $\left|x-x_{0}\right| \leq h$.

This theorem is proved by showing that the sequence of functions, $y_{n}(x)$, defined by

$$
\begin{aligned}
& y_{0}(x)=y_{0}, \\
& y_{n}(x)=y_{0}+\int_{x_{0}}^{x} f\left(t, y_{n-1}(t)\right) d t,
\end{aligned}
$$

converges to a function $y(x)$, which is a continuous solution of (48) and furthermore, the only continuous solution.

Note 1. The proof will also go through for any initial iterate, $y_{0}(x)$, which is continuous on R. In our applications, we use the solution to the unperturbed system as the initial iterate.

\section{C.2. Existence and Uniqueness of Mean Value Approximant for Perturbed System}

In this section, we demonstrate that the approximate Picard iterations (where each Picard iterate is evaluated using the mean value approximations of Section 3.3) converge to a solution $\hat{y}$ which is the unique continuous solution to (50) in the limit as $x$ approaches $x_{0}$. In our applications, the approximant to the mean value will be the same for each iterate. We will demonstrate the result in this case.

Theorem 3. Let $f_{u}(x, y)$ and $f_{p}(x, y)$ be continuous on $R: x_{0} \leq x \leq x_{0}+a$, $\left|y-y_{0}\right| \leq b$, and furthermore let $f_{p}(x, y)$ be Lipschitz on $R$. There exists a number $h>0$ with the property that the initial value problem

$$
y^{\prime}=f_{p}(x, y) \quad y\left(x_{0}\right)=y_{0}
$$

has one and only one solution, $y=y(x)$, on the interval $\left|x-x_{0}\right| \leq h$. Moreover, the sequence of functions

$$
\begin{aligned}
& \hat{y}_{0}(x)=y_{0}+f_{u}\left(\hat{\xi}_{x}, y_{0}\right)\left(x-x_{0}\right), \\
& \hat{y}_{n}(x)=y_{0}+f_{p}\left(\hat{\xi}_{x}, \hat{y}_{n-1}\left(\hat{\xi}_{x}\right)\right)\left(x-x_{0}\right),
\end{aligned}
$$

converges to $y(x)$ as $x$ approaches $x_{0}$.

Proof. Since both $f_{u}$ and $f_{p}$ are continuous on $R$, a closed and bounded set, there exists an $M$ such that both

$$
\left|f_{u}\right| \leq M \quad \text { and } \quad\left|f_{p}\right| \leq M
$$

Furthermore, since $f_{p}$ is Lipschitz on $R$, there exists a $K$ such that

$$
\left|f_{p}\left(x, y_{1}\right)-f_{p}\left(x, y_{2}\right)\right| \leq K\left(\left|y_{1}-y_{2}\right|\right)
$$

for all $\left(x, y_{1}\right)$ and $\left(x, y_{2}\right)$ in $R$. 
Now, select $h$ such that both

$$
K h<1
$$

and

$$
R^{\prime} \subset R \quad \text { where } \quad R^{\prime}=\left\{(x, y)|| x-x_{0}|\leq h,| y-y_{0} \mid \leq M h\right\} .
$$

To prove this theorem, it is necessary to show (i) the solution $y(x)$ to (50) exists and is unique, (ii) the sequence of functions $\hat{y}_{n}$ converges uniformly to a function $\hat{y}$, and (iii) $\hat{y}$ converges to $y(x)$ for $x$ close to $x_{0}$.

In this case, (i) follows from traditional Picard-Lindelöf results [18], [41]. Now we turn our attention to (ii).

Claim 1. Each iterate $\hat{y}_{n}$ lies in $R^{\prime}$.

By definition $\left(x_{0}, y_{0}\right) \subset R^{\prime}$. Additionally, if $\left(x, y_{0}\right) \subset R^{\prime}$, then $\left(\hat{\xi}_{x}, y_{0}\right) \subset R^{\prime}$. As defined, $\hat{y}_{0}(x)=y_{0}+f_{u}\left(\hat{\xi}_{x}, y_{0}\right)\left(x-x_{0}\right)$. It follows that

$$
\begin{aligned}
\left|\hat{y}_{0}(x)-y_{0}\right| & =\left|f_{u}\left(\hat{\xi}_{x}, y_{0}\right)\left(x-x_{0}\right)\right| \\
& =\left|f_{u}\left(\hat{\xi}_{x}, y_{0}\right)\right|\left|x-x_{0}\right| \\
& \leq M h .
\end{aligned}
$$

So, $\hat{y}_{0}(x) \subset R^{\prime}$.

Now assume $\hat{y}_{n-1}(x) \subset R^{\prime}$.

Then, since $\hat{y}_{n}(x)=y_{0}+f_{p}\left(\hat{\xi}_{x}, \hat{y}_{n-1}\left(\hat{\xi}_{x}\right)\right)\left(x-x_{0}\right)$, we have

$$
\begin{aligned}
\left|\hat{y}_{n}(x)-y_{0}\right| & =\left|f_{p}\left(\hat{\xi}_{x}, \hat{y}_{n-1}\right)\left(x-x_{0}\right)\right| \\
& =\left|f_{p}\left(\hat{\xi}_{x}, \hat{y}_{n-1}\right)\right|\left|x-x_{0}\right| \\
& \leq M h .
\end{aligned}
$$

It follows that $\hat{y}_{n}(x) \subset R^{\prime}$. Therefore, by induction Claim 1 is true.

Showing that the sequence $\hat{y}_{n}(x)$ converges is identical to showing the convergence of the sum

$$
\Sigma_{y}=\hat{y}_{0}(x)+\sum_{n=1}^{\infty}\left[\hat{y}_{n}(x)-\hat{y}_{n-1}(x)\right],
$$

since by definition the $i^{t h}$ partial sum is equal to $\hat{y}_{i}(x)$.

Since $f_{u}$ and $f_{p}$ are both continuous on $R$, it follows that $\hat{y}_{0}(x)$ and $\hat{y}_{1}(x)$ are both continuous on $R$, and consequently that $\hat{y}_{1}(x)-\hat{y}_{0}(x)$ is continuous on $R^{\prime}$. Since $R^{\prime}$ is a closed and bounded set, there exists an $a$ such that

$$
a=\max _{x \in R^{\prime}}\left|\hat{y}_{1}(x)-\hat{y}_{0}(x)\right|
$$

Claim 2. For $x \in R^{\prime},\left|\hat{y}_{n+1}(x)-\hat{y}_{n}(x)\right| \leq a(K h)^{n}$. 
Clearly by definition of $a$, this is true for $n=0$.

Assume that $\left|\hat{y}_{n}(x)-\hat{y}_{n-1}(x)\right| \leq a(K h)^{(n-1)}$ and consider $\left|\hat{y}_{n+1}(x)-\hat{y}_{n}(x)\right|$,

$$
\begin{aligned}
\left|\hat{y}_{n+1}(x)-\hat{y}_{n}(x)\right| & =\left|\left(f_{p}\left(\hat{\xi}_{x}, \hat{y}_{n}\left(\hat{\xi}_{x}\right)\right)-f_{p}\left(\hat{\xi}_{x}, \hat{y}_{n-1}\left(\hat{\xi}_{x}\right)\right)\right)\left(x-x_{0}\right)\right| \\
& =\left|f_{p}\left(\hat{\xi}_{x}, \hat{y}_{n}\left(\hat{\xi}_{x}\right)\right)-f_{p}\left(\hat{\xi}_{x}, \hat{y}_{n-1}\left(\hat{\xi}_{x}\right)\right)\right|\left|\left(x-x_{0}\right)\right| \\
& \leq K\left|\hat{y}_{n}\left(\hat{\xi}_{x}\right)-\hat{y}_{n-1}\left(\hat{\xi}_{x}\right)\right|\left|\left(x-x_{0}\right)\right| \\
& \leq K\left(a(K h)^{n-1}\right) h \\
& \leq a(K h)^{n} .
\end{aligned}
$$

Thus, Claim 2 is shown by induction.

Each term in the series $\Sigma_{y}$ is less than or equal to each corresponding term in the series

$$
\left|\max _{x \in R^{\prime}} \hat{y}_{0}(x)\right|+\sum_{n=1}^{\infty} a(K h)^{n-1},
$$

which converges by assumption (54). Consequently, $\Sigma_{y}$ converges uniformly to a function $\hat{y}(x)$ [35]. It follows by definition of $\Sigma_{y}$ that the sequence of functions $\hat{y}_{n}(x)$ converges uniformly to $\hat{y}(x)$, proving (ii).

It remains to show (iii). We will demonstrate this by showing that $\hat{y}(x)$ is the unique continuous solution to (50) as $x$ approaches $x_{0}$. Equivalently, this can be stated as

$$
\lim _{x \rightarrow x_{0}}\left(\hat{y}(x)-y_{0}-\int_{x_{0}}^{x} f_{p}(t, \hat{y}(t)) d t\right)=0 .
$$

By definition

$$
\hat{y}_{n}(x)-y_{0}-f_{p}\left(\hat{\xi}_{x}, y_{n-1}\left(\hat{\xi}_{x}\right)\right)\left(x-x_{0}\right)=0
$$

Therefore,

$$
\begin{gathered}
\hat{y}(x)-y_{0}-\int_{x_{0}}^{x} f_{p}(t, \hat{y}(t)) d t=\hat{y}(x)-\hat{y}_{n}(x)+f_{p}\left(\hat{\xi}_{x}, y_{n-1}\left(\hat{\xi}_{x}\right)\right)\left(x-x_{0}\right) \\
-\int_{x_{0}}^{x} f_{p}(t, \hat{y}(t)) d t .
\end{gathered}
$$

Letting the mean value of $f_{p}$ be given by $\xi_{x}$, we have

$$
\begin{aligned}
\hat{y}(x)-y_{0}-\int_{x_{0}}^{x} f_{p}(t, \hat{y}(t)) d t= & \hat{y}(x)-\hat{y}_{n}(x) \\
& +\left(f_{p}\left(\hat{\xi}_{x}, \hat{y}_{n-1}\left(\hat{\xi}_{x}\right)\right)-f_{p}\left(\xi_{x}, \hat{y}\left(\xi_{x}\right)\right)\right)\left(x-x_{0}\right) .
\end{aligned}
$$

Taking the absolute value on each side and using the triangle inequality, we find

$$
\begin{aligned}
& \left|\hat{y}(x)-y_{0}-\int_{x_{0}}^{x} f_{p}(t, \hat{y}(t)) d t\right| \\
& \quad \leq\left|\hat{y}(x)-\hat{y}_{n}(x)\right|+\left|f_{p}\left(\hat{\xi}_{x}, \hat{y}_{n-1}\left(\hat{\xi}_{x}\right)\right)-f_{p}\left(\xi_{x}, \hat{y}\left(\xi_{x}\right)\right)\right|\left|x-x_{0}\right|
\end{aligned}
$$




$$
\begin{aligned}
\leq & \left|\hat{y}(x)-\hat{y}_{n}(x)\right|+\left|f_{p}\left(\hat{\xi}_{x}, \hat{y}_{n-1}\left(\hat{\xi}_{x}\right)\right)-f_{p}\left(\hat{\xi}_{x}, \hat{y}\left(\hat{\xi}_{x}\right)\right)\right|\left|x-x_{0}\right| \\
& +\left|f_{p}\left(\hat{\xi}_{x}, \hat{y}\left(\hat{\xi}_{x}\right)\right)-f_{p}\left(\xi_{x}, \hat{y}\left(\xi_{x}\right)\right)\right|\left|x-x_{0}\right| \\
\leq & \left|\hat{y}(x)-\hat{y}_{n}(x)\right|+K\left|\hat{y}_{n-1}\left(\hat{\xi}_{x}\right)-\hat{y}\left(\hat{\xi}_{x}\right)\right|\left|x-x_{0}\right| \\
& +\left|f_{p}\left(\hat{\xi}_{x}, \hat{y}\left(\hat{\xi}_{x}\right)\right)-f_{p}\left(\xi_{x}, \hat{y}\left(\xi_{x}\right)\right)\right|\left|x-x_{0}\right| .
\end{aligned}
$$

By uniform convergence, the first two terms can be chosen arbitrarily small by simply selecting $n$ large enough. The last term represents the error between the exact integral and its mean value approximant. By Theorem 1 it can also be made arbitrarily small by letting $x$ approach $x_{0}$. Therefore, the right-hand side and, consequently, the left-hand side go to zero as $n$ grows large and $x$ becomes sufficiently close to $x_{0}$, implying that $\hat{y}(x)$ is the solution to (50) in the limit.

\section{Appendix D. Iteration Order-Does It Matter?}

Since this iteration procedure is being used to "solve" a Hamiltonian system, it is desirable for each iteration step to preserve the constant of motion, $\dot{H}=0$.

Consider the general Hamiltonian system, $H(y, z)$, where $y$ and $z$ represent a particular partition of the state. Since $H$ is constant, assume $H(y, z)=E_{0}$. Let $z=H^{-1}(y)$ be defined such that $H\left(y, H^{-1}(y)\right)=E_{0}$. Then clearly $\frac{d H\left(y, H^{-1}(y)\right)}{d t}=0$.

Consider the iterative approach,

$$
\begin{aligned}
& y_{(n+1)}=y_{0}+\int_{t_{0}}^{t} f\left(y_{n}, z_{n}\right) d \tau, \\
& z_{(n+1)}=H^{-1}\left(y_{(n+1)}\right) .
\end{aligned}
$$

In this case the energy of the $(n+1)^{\text {th }}$ iterate is given by

$$
\begin{aligned}
H_{(n+1)} & =H\left(y_{(n+1)}, z_{(n+1)}\right) \\
& =H\left(y_{(n+1)}, H^{-1}\left(y_{(n+1)}\right)\right) \\
& =E_{0} .
\end{aligned}
$$

Hence the iteration scheme given in (67), in which the new iterate for $y$ is formed using the old iterates for $y$ and $z$ and the new iterate for $z$ is formed using the new iterate of $y$, is conservative.

However, the following iteration scheme,

$$
\begin{aligned}
& y_{(n+1)}=y_{0}+\int_{t_{0}}^{t} f\left(y_{n}, z_{n}\right) d \tau, \\
& z_{(n+1)}=H^{-1}\left(y_{n}\right),
\end{aligned}
$$

in which each iterate is defined using only the previous iterate, is not conservative; that is, each iterate does not maintain the Hamiltonian property, $\dot{H}=0$. In particular, the energy of the $(n+1)^{t h}$ iterate is given by

$$
\begin{aligned}
H_{(n+1)} & =H\left(y_{(n+1)}, z_{(n+1)}\right) \\
& =H\left(y_{(n+1)}, H^{-1}\left(y_{n}\right)\right),
\end{aligned}
$$

which in general will not be equal to $E_{0}$. 
The above derivation suggests that the key determinant of the conservative nature of the iteration procedure is whether any old iterates are used in the construction of $z_{(n+1)}$. If so, the iteration scheme is not conservative.

The "accelerated" iteration scheme (74) treats each iteration in a sequential manner and uses the most recent approximant in each successive step of the current iterate. By assigning $\hat{p}_{r_{n}}\left(q_{r}\right)=H_{p e r t}^{-1}\left(q_{r}, \hat{q_{\theta}(n-1)}\left(q_{r}\right), \hat{p}_{\theta n}\left(q_{r}\right)\right)$, it violates the principle discussed above, implying that $\dot{H} \neq 0$. Nevertheless, this accelerated approach may prove useful because it was found to produce better mean errors after one iteration than the other approaches presented in Appendix F.

\section{Appendix E. Approximation Error Data Collection Process}

This appendix details the simulation procedure used to obtain the data presented in Figures 6 and 9. All simulations are run in Mathematica and are done for the same set of initial conditions.

For these figures, initial conditions are taken in bottom coordinates and are selected to guarantee a symmetric stance, $\theta_{b}=0, p_{r b}=0$. It should be noted that the restriction to a symmetric stance is for convenience and is not required for the approximation strategy presented in Section 4. In this work, initial conditions are selected from a cube of the form $r_{b} \in[0.75,0.975] \mathrm{m}, p_{\theta b} \in[1.5,6.5] \mathrm{kg} \mathrm{m}^{2} \mathrm{rad} / \mathrm{s}$, and $U\left(r_{b}\right) \in$ $[2.5,7.5] \mathrm{kg} \mathrm{m}^{2} / \mathrm{s}^{2}$ for the perturbed case. ${ }^{15}$ To explore this cube, ten equally spaced points in each interval are selected, yielding the 1000 point grid of initial conditions shown in Figure 7(a).

The SLIP stance dynamics are numerically integrated using Mathematica's NDSolve routine condition. In each case, the liftoff condition is chosen as $q_{r l}=1 \mathrm{~m}$, and the time at which this occurs is obtained numerically using the Mathematica's FindRoot routine. Various liftoff (time, angle, radial and angular momentum) and apex (hopping height, velocity, and duty factor) parameters are read off from these numerical solutions and compared to their appropriate counterpart generated using the approximation method of Section 4. The comparison is undertaken in terms of the percent error (PE), shown here for the case of the apex hopping height,

$$
P E=100 \frac{\left\|y_{a}-\hat{y}_{a}\right\|_{2}}{\left\|y_{a}\right\|_{2}} .
$$

After computing these errors for each initial condition, the mean and maximum percent errors are calculated as shown below and reported in the figures.

$$
\operatorname{Mean} P E=\frac{1}{N} \sum_{i=1}^{N} P E_{i}
$$

and

$$
\operatorname{Max} P E=\max _{N} P E_{i}
$$

\footnotetext{
${ }^{15}$ For the unperturbed case, the cube of initial conditions is selected as $r_{b} \in[0.75,0.975] m, p_{\theta b} \in$ $[1.5,6.5] \mathrm{kg} \mathrm{m}^{2} \mathrm{rad} / \mathrm{s}$, and $U\left(r_{b}\right) \in[0.25,6.25] \mathrm{kg} \mathrm{m}^{2} / \mathrm{s}^{2}$.
} 


\section{Appendix F. Alternate "Simple" Solutions}

For applications it will be desirable to generate sufficiently good approximants in a minimum number of steps, since each increasing iterate increases in functional complexity. A larger number of iterates may be acceptable for spring identification because it primarily involves evaluation of the functions and their derivatives. Since the iterates are constructed by a number of function compositions, both the iterates and the derivatives will have a reasonable amount of structure and will be "easy" to evaluate numerically.

For purposes of control, where parametric insight is necessary to achieve the desired control objectives, more than one iterate may not be feasible. In addition to being tractable analytically, it is also required that the iterate give "sufficiently" small errors. In this light, it may prove useful, for applications purposes, to generate a first iterate which is "better" than (33). We propose two alternative solutions. One is "better" in that is generates smaller mean errors, and the other is better in that it is analytically simpler.

The first approach is an "accelerated" iteration method. It is motivated by a consideration of the effects of reintroducing gravity during stance. There are two major effects of the gravitational perturbation during stance. The first is that angular momentum is no longer constant. The second is that while total energy is still constant, its functional form changes from $H_{\text {unpert }}$ to $H_{\text {pert }}$.

"Accelerated" Iterate. The idea in the accelerated iteration is to first account for the presence of gravity by modifying $p_{\theta}$ and $p_{r}$ and then use these solutions to generate $q_{\theta}$ and $t_{s}$. This strategy is shown in (74). We begin by correcting $p_{\theta}$. That is, we first generate the iterate $\hat{p}_{\theta 1}$ using the unperturbed solution, $\hat{q}_{\theta 0}\left(q_{r}\right)$ and $\hat{p}_{r 0}\left(q_{r}\right)=H_{u n p e r t}^{-1}\left(q_{r}, \hat{p}_{\theta 0}\left(q_{r}\right)\right)$. Then this updated approximant, $\hat{p}_{\theta 1}$, along with the old approximant for $\hat{q}_{\theta 0}\left(q_{r}\right)$ is used to adjust the total energy by $\hat{p}_{r 1}\left(q_{r}\right)=H_{p e r t}^{-1}\left(q_{r}, \hat{q}_{\theta 0}\left(q_{r}\right) \hat{p}_{\theta 1}\left(q_{r}\right)\right)$. Having adjusted $p_{r}$ and $p_{\theta}$ to account for gravity, we use these solutions to generate the $\hat{t}_{s 1}\left(q_{r}\right)$ and $\hat{q}_{\theta 1}\left(q_{r}\right)$ iterates, resulting in

$$
\begin{aligned}
\hat{p}_{\theta 1}\left(q_{r}\right)= & p_{\theta b}+\frac{m^{2} g \hat{\xi}_{r} \sin \left(\hat{q}_{\theta 0}\left(\hat{\xi}_{r}\right)\right)}{H_{\text {unpert }}^{-1}\left(\hat{\xi}_{r}, \hat{p}_{\theta 0}\left(\hat{\xi}_{r}\right)\right)}\left(q_{r}-r_{b}\right), \\
\hat{p}_{r 1}\left(q_{r}\right)= & H_{\text {pert }}^{-1}\left(q_{r}, \hat{q}_{\theta 0}\left(q_{r}\right), \hat{p}_{\theta 1}\left(q_{r}\right)\right) \\
= & {\left[2 m\left[\left(U\left(r_{b}\right)-U\left(q_{r}\right)\right)+m g\left(r_{b} \cos \left(\theta_{b}\right)-q_{r} \cos \left(\hat{q}_{\theta 0}\left(q_{r}\right)\right)\right)\right]\right.} \\
& \left.+\left(\frac{p_{\theta b}^{2}}{r_{b}^{2}}-\frac{\hat{p}_{\theta 1}^{2}\left(q_{r}\right)}{q_{r}^{2}}\right)\right]^{\frac{1}{2}} \\
\hat{t}_{s 1}\left(q_{r}\right)= & t_{s b}+\frac{m}{\hat{p}_{r 1}\left(\hat{\xi}_{r}\right)}\left(q_{r}-r_{b}\right), \\
\hat{q}_{\theta 1}\left(q_{r}\right)= & \theta_{b}+\frac{p_{\theta 1}\left(\hat{\xi}_{r}\right)}{\hat{\xi}_{r}^{2} \hat{p}_{r 1}\left(\hat{\xi}_{r}\right)}\left(q_{r}-r_{b}\right) .
\end{aligned}
$$

We call this approach "accelerated" because the most recent updates for $\hat{p}_{\theta 1}$ and $\hat{p}_{r 1}$ 
are used in the generation of the other iterates. This has the disadvantage of not preserving energy, since the update $\hat{p}_{r_{1}}\left(q_{r}\right)$ is constructed using $\hat{p}_{\theta 1}$ and $\hat{q}_{\theta 0}$ (see Appendix D).

Linearized Perturbation. The second approach is not an iteration scheme, but rather the solution to a problem somewhere between the perturbed and unperturbed solution. In this solution we linearize the gravitational perturbation about $q_{\theta}=0$. Then the Hamiltonian becomes

$$
H_{\text {mod }}=H_{\text {unpert }}+H_{\text {lin }}
$$

where

$$
H_{\text {unpert }}=\frac{1}{2 m}\left(p_{r}^{2}+\frac{p_{\theta}^{2}}{q_{r}^{2}}\right)+U\left(q_{r}\right) \quad \text { and } \quad H_{\text {lin }}=m g q_{r} .
$$

Then the vector field, $X_{H_{m o d}}$, arising from the Hamiltonian, $H_{m o d}$, is given by

$$
\left[\begin{array}{c}
\dot{q}_{r} \\
\dot{q}_{\theta} \\
\dot{p}_{r} \\
\dot{p}_{\theta}
\end{array}\right]=X_{H_{m o d}}(\boldsymbol{q}, \boldsymbol{p})=\left[\begin{array}{c}
\frac{p_{r}}{m_{\theta}} \\
\frac{p_{\theta}}{m q_{r}^{2}} \\
\frac{p_{\theta}^{2}}{m q_{r}^{3}}-D U\left(q_{r}\right)-m g \\
0
\end{array}\right] .
$$

Notice that these also are the dynamics of a central force problem, albeit slightly different from those in (4). As such, we can write down the integrals and apply the MVT as before. The results are

$$
\begin{aligned}
& p_{\theta}\left(q_{r}\right)=p_{\theta b}, \\
& p_{r}\left(q_{r}\right)=\left[p_{\theta b}^{2}\left(\frac{1}{r_{b}^{2}}-\frac{1}{q_{r}^{2}}\right)+2 m\left(\left(U\left(r_{b}\right)-U\left(q_{r}\right)\right)-m g\left(q_{r}-r_{b}\right)\right)\right]^{\frac{1}{2}}, \\
& \hat{q}_{\theta}\left(q_{r}\right)=\frac{p_{\theta b}\left(q_{r}-r_{b}\right)}{\hat{\xi}_{r}^{2}\left[p_{\theta b}^{2}\left(\frac{1}{r_{b}^{2}}-\frac{1}{\hat{\xi}_{r}^{2}}\right)+2 m\left(\left(U\left(r_{b}\right)-U\left(\hat{\xi}_{r}\right)\right)-m g\left(\hat{\xi}_{r}-r_{b}\right)\right)\right]^{\frac{1}{2}}}, \\
& \hat{t}_{s}\left(q_{r}\right)=\frac{m\left(q_{r}-r_{b}\right)}{\left[p_{\theta b}^{2}\left(\frac{1}{r_{b}^{2}}-\frac{1}{\hat{\xi}_{r}^{2}}\right)+2 m\left(\left(U\left(r_{b}\right)-U\left(\hat{\xi}_{r}\right)\right)-m g\left(\hat{\xi}_{r}-r_{b}\right)\right)\right]^{\frac{1}{2}}} .
\end{aligned}
$$

This approximate solution is the simplest-it is not even an iterate, but rather the solution to a modified central force problem. However, this solution is possible only because of constant angular momentum in the central force problem. Hence is it farther from the physical reality of the perturbed problem than the iterations of (33) and (74) which account for changes in angular momentum.

Figure 9 shows the errors arising from these three different "simple" solutions. ${ }^{10}$ The data in this figure reveals no clear cut winner-there seems to be a tradeoff in performance between certain parameters. For example, both the accelerated and the linearized perturbation do better than the unaccelerated iterate for $t_{s}, q_{\theta}, y_{a}$, and $\beta$ while doing worse for $p_{r}, p_{\theta}$, and $\dot{x}_{a}$. Even so, the accelerated iterate appears to do better on average. That is, while some of the maximum errors may be higher, the mean errors are roughly the same or better than the other two. However this comes at the cost of a slight 

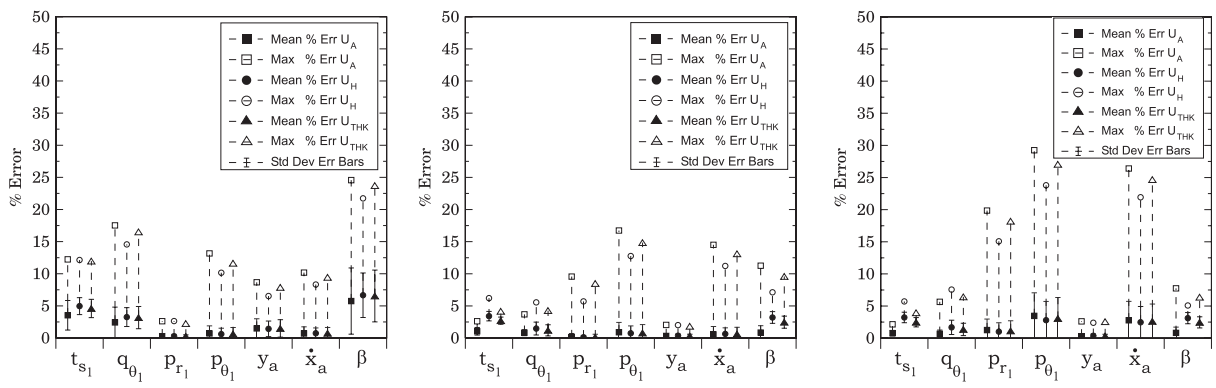

Fig. 9. Errors in liftoff and apex parameters in the approximate iterates when compared to the "true" simulated values of the perturbed system: (left) first iterate (unaccelerated, conservative) (33); (middle) first iterate (accelerated, not conservative) (74); (right) Modified central force solution (linearized perturbation) (77). Errors are computed for three springs introduced in Section 3.2, i.e. the compressed air spring, $U_{A}\left(q_{r}\right)$, the Hooke's Law Spring, $U_{H}\left(q_{r}\right)$, and the effective spring arising from a Torsional Hooke's Law Spring at the knee, $U_{T H K}\left(q_{r}\right)$.

increase in functional complexity. On the other hand, while the linearized perturbation approach has some of the worst maximum errors, ${ }^{16}$ it offers the simplest functional form.

\section{Acknowledgments}

We are indebted to Tony Bloch and Phil Holmes for numerous tutorial discussions. Additionally, we would like to thank Phil Holmes for a number of helpful suggestions regarding the SLIP perturbed dynamics and Charles Doering for suggestions regarding the mean value approximations. This work was supported in part by National Science Foundation Grant IRI-9612357.

\section{References}

1. R. M. Alexander. Three uses for springs in legged locomotion. International Journal of Robotics Research, 9(2):53-61, 1990.

2. R. M. Alexander and A. S. Jayes. Vertical movement in walking and running. Journal of Zoology, London, 185:27-40, 1978.

3. N. Bernstein. The Co-ordination and Regulation of Movements. Pergamon Press, Oxford, 1967.

4. R. Blickhan. The spring-mass model for running and hopping. Journal of Biomechanics, 22:1217-1227, 1989.

5. R. Blickhan and R. J. Full. Similarity in multilegged locomotion: Bouncing like a monopode. Journal of Comparative Physiology A, 173:509-517, 1993.

\footnotetext{
${ }^{16}$ The linearized perturbation is integrable because it maintains angular momentum as a constant of motion. This helps explain why the largest errors are observed in $p_{\theta}$ and $\dot{x}_{a}$.
} 
6. P. H. Channon, S. H. Hopkins, and D. T. Pham. A variational approach to the optimization of gait for a bipedal robot. Proceedings of the Institution of Mechanical Engineers, 210(C2):177186, 1996.

7. C. Chevallereau, A. Formal'sky, and B. Perrin. Control of a walking robot with feet following a reference trajectory derived from ballistic motion. In Proceedings of the IEEE International Conference on Robotics and Automation, pages 1094-1099, Albuquerque, NM, April 1997.

8. C. T. Farley, J. Glasheen, and T. A. McMahon. Running springs: Speed and animal size. Journal of Experimental Biology, 185:71-86, 1993.

9. D. Fortin, J. F. Lamy, and D. Thalman. A multiple track animator system for motion synchronization. In N. I. Badler and J. K. Tsotsos, editors, Motion: Representation and Perception: Proceedings of the ACM SIGGRAPH/SIGART Interdisciplinary Workshop on Motion, Representation and Perception, held in Toronto, Ontario, Canada, 1983, pages 303-310.

10. R. J. Full. Integration of individual leg dynamics with whole body movement in arthropod locomotion. In R. D. Beer, R. E. Ritzmann, and T. McKenna, editors, Biological Neural Networks in Invertebrate Neuroethology and Robotics, pages 3-20. Academic Press, San Diego, CA, 1993.

11. R. J. Full and M. S. Tu. Mechanics of six-legged runners. Journal of Experimental Biology, 148:129-146, 1990.

12. R. J. Full, M. S. Tu, and L. H. Ting. Dynamics of insect locomotion compared to hexapod walking machines. Proceedings of the American Society of Mechanical Engineering, DSC 17:35-39, 1989.

13. C. M. Ginsberg and D. Maxwell. Graphical marionette. In N. I. Badler and J. K. Tsotsos, editors, Motion: Representation and Perception: Proceedings of the ACM SIGGRAPH/SIGART Interdisciplinary Workshop on Motion, Representation and Perception, held in Toronto, Ontario, Canada, 1983, pages 303-310. 1983.

14. H. Goldstein. Classical Mechanics. Addison-Wesley, Reading, MA, 2nd edition, 1980.

15. P. Gregorio, M. Ahmadi, and M. Buehler. Experiments with an electrically actuated planar hopping robot. In T. Yoshikawa and F. Miyazaki, editors, "Experimental Robotics III," Lecture Notes in Control and Information Sciences 200, pages 269-281. Springer-Verlag, New York, 1994.

16. J. W. Grizzle, G. Abba, and F. Plestan. Asymptotically stable walking for biped robots: Analysis via systems with impulse effects. IEEE Transactions on Automatic Control. To Appear.

17. J. W. Grizzle, G. Abba, and F. Plestan. Poincaré's method for systems with impulse effects: Application to mechanical biped locomotion. In IEEE Conference on Decision and Control, Phoenix, AZ, December 1999.

18. P. Hartman. Ordinary Differential Equations. Birkhäuser, Boston, 2nd edition, 1982.

19. P. Holmes. Poincaré, celestial mechanics, dynamical systems theory and "chaos." Physics Reports (Review Section of Physics Letters), 193(3):137-163, 1990.

20. Y. Hurmuzlu. Dynamics of bipedal gait: Part I-Objective functions and the contact event of a planar five-link biped. ASME Journal of Applied Mechanics, 60(2):331-336, 1993.

21. Y. Hurmuzlu. Dynamics of bipedal gait: Part II-Stability analysis of a planar five-link biped. ASME Journal of Applied Mechanics, 60(2):337-343, 1993.

22. D. E. Koditschek and M. Bühler. Analysis of a simplified hopping robot. International Journal of Robotics Research, 10(6):587-605, December 1991.

23. T.-T. Lee and J.-H. Liao. Trajectory planning and control of a 3-link biped robot. In Proceedings of the IEEE International Conference On Robotics and Automation, pages 820-823, Philadelphia, PA, April 1988.

24. A. J. Lichtenberg and M. A. Lieberman. Regular and Stochastic Motion. Springer-Verlag, New York, 1983.

25. T. McGeer. Passive dynamic walking. International Journal of Robotics Research, 9(2):62-82, 1990.

26. R. B. McGhee. Robot locomotion. In R. N. Herman, S. Grillner, P. S. Stein, and D. G. Stuart, editors, Neural Control of Locomotion. Plenum Press, New York, 1976. 
27. R. T. M'Closkey and J. W. Burdick. Periodic motions of a hopping robot with vertical and forward motion. International Journal of Robotics Research, 12(3):197-218, 1993.

28. T. A. McMahon. Role of compliance in mammalian running gaits. Journal of Experimental Biology, 115:263-282, 1985.

29. T. A. McMahon, G. Valiant, and E. C. Frederick. Groucho running. Journal of Applied Physiology, 62(6):2326-2337, 1987.

30. J. Pratt, P. Dilworth, and G. Pratt. Virtual model control of a bipedal walking robot. In Proceedings of the IEEE International Conference On Robotics and Automation, pages 193-198, Albuquerque, NM, April 1997.

31. H. Rad, P. Gregorio, and M. Buehler. Design, modeling and control of a hopping robot. In Proceedings of the IEEE/RSJ Conference On Intelligent Systems and Robots, pages 17781785, Yokohama, Japan, July 1993.

32. M. H. Raibert. Legged Robots That Balance. MIT Press, Cambridge, MA, 1986.

33. A. A. Rizzi and D. E. Koditschek. Active visual estimator for dexterous manipulation. IEEE Transactions on Robotics and Automation, 12(5):697-713, October 1996.

34. M. Rostami and G. Bessonnet. Impactless sagittal gait of a biped robot during the single support phase. In Proceedings of the IEEE International Conference On Robotics and Automation, pages 1385-1391, Leuven, Belgium, May 1998.

35. W. Rudin. Principles of Mathematical Analysis. McGraw-Hill, New York, 3rd edition, 1976.

36. U. Saranli, W. J. Schwind, and D. E. Koditschek. Toward the control of a multi-jointed, monoped runner. In Proceedings of the IEEE International Conference On Robotics and Automation, pages 2676-2682, Leuven, Belgium, May 1998.

37. W. J. Schwind. Spring Loaded Inverted Pendulum Running: A Plant Model. PhD thesis, University of Michigan, Ann Arbor, 1998.

38. W. J. Schwind, J. Ji, and D. E. Koditschek. A physically motivated further note on the mean value theorem for integrals. American Mathematical Monthly, 106(6):559-564, June-July 1999.

39. W. J. Schwind and D. E. Koditschek. Control of forward velocity for a simplified planar hopping robot. In Proceedings of the IEEE International Conference on Robotics and Automation, Nagoya, Japan, May 1995.

40. W. J. Schwind and D. E. Koditschek. Characterization of monoped equilibrium gaits. In Proceedings of the IEEE International Conference on Robotics and Automation, pages 19861992, Albuquerque, NM, April 1997.

41. G. F. Simmons. Differential Equations with Applications and Historical Notes. McGraw-Hill, New York, 1972.

42. A. Takanishi, M. Ishida, Y. Yamazaki, and I. Kato. The realizaion of dynamic walking by the biped walking robot WL-10RD. In Proceedings of '85 International Conference on Advanced Robotics, pages 459-466, Tokyo, Japan, September 1985.

43. A. F. Vakakis, J. W. Burdick, and T. K. Caughey. An 'interesting' strange attractor in the dynamics of a hopping robot. International Journal of Robotics Research, 10(6):606-618, December 1991.

44. E. T. Whittaker. A Treatise on the Analytical Dynamics of Particles and Rigid Bodies. Cambridge University Press, New York, 4th edition, 1904.

45. M. Yamada, J. Furusho, and A. Sano. Dynamic control of walking robot with kick-action. In Proceedings of '85 International Conference on Advanced Robotics, pages 405-412, Tokyo, Japan, September 1985.

46. Y.F. Zheng, J. Shen, and F. R. Sias. A motion control scheme for a biped robot to climb sloping surfaces. In Proceedings of the IEEE International Conference On Robotics and Automation, pages 814-816, Philadelphia, PA, April 1988. 